\title{
EXISTENCE THEOREMS FOR PARETO OPTIMIZATION; MULTIVALUED AND BANACH SPACE VALUED FUNCTIONALS ${ }^{1}$
}

\author{
BY
}

\author{
L. CESARI AND M. B. SURYANARAYANA
}

\begin{abstract}
Existence theorems are obtained for optimization problems where the cost functional takes values in an ordered Banach space. The order is defined in terms of a closed convex cone in the Banach space; and in this connection, several relevant properties of cones are studied and they are shown to coincide in the finite dimensional case. The notion of a weak (Pareto) extremum of a subset of an ordered Banach space is then introduced. Existence theorems are proved for extrema for Mayer type as well as Lagrange type problems-in a manner analogous to and including those with scalar valued cost. The side conditions are in the form of general operator equations on a class of measurable functions defined on a finite measure space. Needed closure and lower closure theorems are proved. Also, several analytic criteria for lower closure are provided. Before the appendix, several illustrative examples are given. In the appendix, a criterion (different from the one used in main text) is given and proved, for the Pareto optimality of an element.
\end{abstract}

1. Introduction. In problems of econometrics the concept of Pareto extremum (maximum, minimum) has recently become relevant. Given a system $I[y]=\left(I_{i}[y], i=1, \ldots, \rho\right)$ of $\rho$ real valued functionals defined on a set $\Omega$, we say that an element $y_{0} \in \Omega$ gives a Pareto minimum to $I[y]$ provided for no element $y \in \Omega, y \neq y_{0}$, it happens that $I_{i}[y] \leqslant I_{i}\left[y_{0}\right], i=1, \ldots, \rho$, with $I_{i}[y]<I_{i}\left[y_{0}\right]$ for at least one $i$. We may say that $y_{0}$ is a Pareto optimal solution (minimum). Analogous definitions hold for maximum and min-max situations.

Since the pioneering work of Pareto [26] in 1896 on the optimization of vector valued functionals, applied to econometrics, there has been considerable research on necessary and sufficient conditions for Pareto optimality, such as by Zadeh, Dacunha and Polak, Olech, Yu, Leitman and $\mathrm{Yu}$, Weinberger and Smale, to name a few.

In the present paper we consider the problem of existence of Pareto optimal solutions.

For the sake of generality, we treat the case of a functional with values in a

Presented to the Society, October 25, 1975 under the title Existence theorems for Pareto optimization problems; received by the editors April 21, 1976.

AMS (MOS) subject classifications (1970). Primary 49A25, 49A10, 49A20, 90A75.

'This research was partially supported by AFOSR grant 71-2122.

- American Mathematical Society 1978 
Banach space $Z$ with an ordering defined by a (closed) convex cone $\Lambda$ in $Z$. Also, we state the problem in the abstract form of optimal control theory with the unknown in a Banach space $X$ and state and control variables in metric spaces $Y$ and $U$ as in [5]. Thus, in particular, the Pareto optimal solutions for finitely many (say, $\rho$ ) functionals of the calculus of variations and of optimal control theory on curves and surfaces are included, with or without side conditions.

We first consider subsets $\mathcal{Q}$ of a Banach space $Z$ which are bounded below in a suitable sense, and we state the concept of weak extremum of $\mathbb{Q}$ in $Z$ with respect to the partial ordering defined by a convex closed cone $\Lambda$ in $Z$. We prove, under hypotheses, on $\mathcal{Q}, Z$ and $\Lambda$, that $\mathscr{Q}$ necessarily possesses at least one weak extremum, which belongs to the weak closure of $\mathcal{Q}$. When $\mathcal{Q}$ is the set of values taken by a given $Z$-valued functional on a set $\Omega$ of admissible elements, then we prove further that at least one weak extremum (optimal value) belongs to $\mathcal{Q}$, that is, an optimal element belongs to $\Omega$. Our existence proofs are based on closure and lower closure theorems similar to those we have previously used in optimal control theory in Banach spaces [5], [6], [12].

We discuss various properties of a convex closed cone $\Lambda$ in a Banach space $Z$, as $\Lambda$ being "pointed", "acute", having property $(\pi)$ and "angle property". We show the interrelationship of these properties in general Banach spaces. They are equivalent properties in finite dimensional spaces. In the rest of the paper we use the angle property. We discuss both Mayer-Pareto type problems in any Banach space $Z$ and Lagrange-Pareto type problems in any reflexive Banach space $Z$. For the sake of clarity, we illustrate our results by considering, as a special case, a Lagrange-Pareto type of problem with finitely many functionals (or $Z=E^{\rho}$ ) and ordinary differential equations, side conditions and controls as usual in optimization theory.

We then analyze the various hypotheses of the main existence theorems, and we show that some of them can be drastically reduced in many practical situations. This analysis is similar to the analogous one for scalar valued functionals in [5]. Also, mere property $(\mathrm{K})$ suffices for the relevant sets instead of property $(\mathrm{Q})$ when dealing with finite dimensional spaces as proved in [34].

2. Cones and orderings in Banach spaces. In this section, we shall recall some relevant definitions and facts regarding cones and orderings defined by cones, in Banach spaces. A detailed study may be found in Bourbaki [3], Day [16], Jameson [17], Klee [20], Yu [30] and others.

Let $Z$ be a real Banach space and $Z^{*}$ be the dual of $Z$. A nonempty subset $\Lambda$ of $Z$ is said to be a convex cone (with vertex at 0 ) if and only if for $\lambda_{1}$, $\lambda_{2} \in \Lambda$ and $a_{1}, a_{2}$ nonnegative real numbers, we have $a_{1} \lambda_{1}+a_{2} \lambda_{2} \in \Lambda$. Some 
authors (Day [16], Jameson [17) use the term "wedge" where we use "convex cone". We shall work with convex cones with at least two elements.

A convex cone $\Lambda$ is "pointed" if and only if $z \in \Lambda$ and $z \in-\Lambda$ implies $z=0$. A cone $\Lambda$ is said to be "acute" provided there is an open half space $L_{a}=\{x \in Z \mid a x>0\}$ with $a \in Z^{*}, a \neq 0$, such that cl $\Lambda \subset L_{a} \cup\{0\}$. A convex cone $\Lambda$ in $Z$ defines an ordering $<$ (or $<_{\Lambda}$ ) in $Z$ as follows: $x<_{\Lambda} y$ if and only if $y-x \in \Lambda$. This ordering is reflexive in the sense that we have $x<_{\Lambda} x$ for every $x \in X$, and transitive in the sense that $x<_{\Lambda} y$ and $y<_{\Lambda} z$ implies $x<_{\Lambda} z$. This ordering is a partial order (or antisymmetric, that is, $x<_{\Lambda} y$ and $y<_{\Lambda} x$ implies $x=y$ ) if and only if $\Lambda$ is pointed.

Given a convex cone $\Lambda$, let $\Lambda^{*}$ denote the "polar" of $\Lambda$, that is, the set $\left\{\eta \in Z^{*} \mid \eta \lambda<0\right.$ for all $\left.\lambda \in \Lambda\right\} \subset Z^{*}$. Clearly, $\Lambda^{*}$ is a closed convex cone in $Z^{*}$. It is of interest to note that, for a closed convex cone $\Lambda, x<_{\Lambda} y$ in $Z$ if and only if $\lambda^{*} x>\lambda^{*} y$ for every $\lambda^{*} \in \Lambda^{*}$. Indeed, if $\left(\Lambda^{*}\right)_{*}=\left\{z \in Z \mid \lambda^{*} z<\right.$ 0 for every $\left.\lambda^{*} \in \Lambda^{*}\right\}$ then $\Lambda \subset\left(\Lambda^{*}\right)_{*}$. Conversely, if there is a $z \in\left(\Lambda^{*}\right)_{*}$ with $z \notin \Lambda$, then by the Hahn-Banach theorem, there is a functional $\eta \in Z^{*}$ with $\eta(z)=1$ and $\eta(\Lambda)=0$. Thus, $\eta \in \Lambda^{*}$ and $z \in\left(\Lambda^{*}\right)_{*}$ and yet $\eta z=1 \nless 0$. This contradiction shows that $\left(\Lambda^{*}\right)_{*} \subset \Lambda$, (and hence $\left.\Lambda=\left(\Lambda^{*}\right)_{*}\right)$. Thus, in particular, it is seen that if $Z$ is reflexive then $\Lambda^{* *}=\Lambda=\left(\Lambda^{*}\right)_{*}$ for any closed convex cone $\Lambda$ in $Z$.

A subset $\mathcal{Q}$ of $Z$ is said to be $\Lambda$-bounded (bounded below) if there is an element $c \in Z$ such that $\mathbb{Q} \subset c+\Lambda=\{c+\lambda \mid \lambda \in \Lambda\}$, that is, $c<_{\Lambda} x$ for all $x \in \mathbb{Q}$.

Given a nonempty subset $\mathcal{Q}$ of $Z$, an element $x_{0} \in Z$ is said to be a (strong) weak $\Lambda$-extremum of $\mathcal{Q}$ if $x_{0}$ belongs to the (strong) weak closure of $\mathbb{Q}\left(\left(x_{0} \in \mathrm{cl}(\mathbb{Q})\right), x_{0} \in \mathrm{W}-\mathrm{cl}(\mathbb{Q})\right)$, and there is no $x \in \mathbb{Q}, x \neq x_{0}$, with $x<_{\Lambda} x_{0}$ (that is, $x_{0}-x \in \Lambda$ ). Thus, $x_{0}$ is a (strong) weak $\Lambda$-extremum of $\mathscr{Q}$ if and only if $x_{0}$ is in the (strong) weak closure of $\mathbb{Q}$, and for every $x \in \mathbb{Q}, x \neq x_{0}$, there is some element $\lambda^{*} \in \Lambda^{*}$ such that $\lambda^{*} x<\lambda^{*} x_{0}\left(\lambda^{*} x_{0} \nless \lambda^{*} x\right)$.

Clearly, weak and strong extrema coincide if $\mathrm{w}-\mathrm{cl}(\mathbb{Q})=\mathrm{s}-\mathrm{cl}(\mathbb{Q})$. This certainly happens if $\mathcal{Q}$ is convex or if $Z$ is finite dimensional and $\mathbb{Q}$ is any subset of $Z$.

We denote by w-Ext ${ }_{\Lambda} \mathscr{Q}$ (or simply $\operatorname{Ext}_{\Lambda} \mathscr{Q}$ ), the set of all weak $\Lambda$-extremum points of $\mathbb{Q}$. Thus, $\operatorname{Ext}_{\Lambda}(\mathbb{Q}) \subset \mathrm{w}-\mathrm{cl}(\mathbb{Q})$. In this paper, we shall consider weak $\Lambda$-extrema only. We shall refer to $\operatorname{Ext}_{\Lambda}(\mathbb{Q})$ also as the set of $\Lambda$-Pareto optimal points of $\mathbb{Q}$.

3. A preliminary lemma. In this section, we shall present a useful criterion for $\Lambda$-Pareto optimality of subsets $\mathcal{Q}$ of a Banach space where $\Lambda$ is a proper convex cone (that is, with at least two elements) in $Z$. For a second criterion, we refer the interested reader to the appendix.

If $\mathcal{Q}$ is a nonempty $\Lambda$-bounded subset of $Z$ and $\lambda^{*} \in \Lambda^{*}$, then the set of 
real numbers $\left\{\mathscr{Z}=\lambda^{*} z \mid z \in \mathbb{Q}\right\}$ is nonempty and bounded above. Indeed, if $\mathbb{Q} \subset c+\Lambda$, then $c<_{\Lambda} z$ and $\lambda^{*} z \leqslant \lambda^{*} c$ for every $z \in \mathbb{Q}$. Let $j=\sup \{\mathscr{Z}=$ $\left.\lambda^{*} z \mid z \in \mathbb{Q}\right\}$.

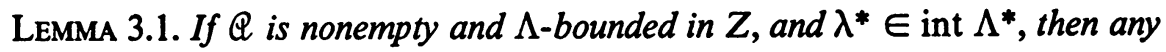
point $z_{0} \in \mathrm{w}-\mathrm{cl}(\mathbb{Q})$, with $\lambda^{*} z_{0}=j$ (if any) is $\Lambda$-Pareto optimal for $\mathbb{Q}$ in $Z$.

Proof. Suppose that $z_{0} \in \mathrm{w}-\mathrm{cl}(\mathbb{Q}), \lambda^{*} z_{0}=j$, and that $z_{0}$ is not Pareto optimal. Then there would be some other point $z \in \mathbb{Q}, z \neq z_{0}$ with $z<_{\Lambda} z_{0}$ or $z_{0}-z \in \Lambda$. Since $\lambda^{*} \in \Lambda^{*}$, we have $\lambda^{*}\left(z_{0}-z\right) \leqslant 0$ or $\lambda^{*} z>\lambda^{*} z_{0}$. But since $z \in \mathbb{Q}, j \geqslant \lambda^{*} z \geqslant \lambda^{*} z_{0}=j$. Hence $\lambda^{*} z_{0}=\lambda^{*} z$ or $\lambda^{*}\left(z_{0}-z\right)=0$.

Since $z_{0}-z \neq 0$, by the Hahn-Banach theorem, there is a linear operator $l \in Z^{*}, l: Z \rightarrow E^{1}$, the reals, with $l\left(z_{0}-z\right)=1$. For $\varepsilon>0$ sufficiently small, then the operator $\lambda_{\varepsilon}=\lambda^{*}+\varepsilon l$ is interior to $\Lambda^{*}$, so that from $z_{0}-z \in \Lambda$ follows $\lambda_{\varepsilon}\left(z_{0}-z\right)<0$. On the other hand $\lambda_{\varepsilon}\left(z_{0}-z\right)=\left(\lambda^{*}+\varepsilon l\right)\left(z_{0}-z\right)=\varepsilon$ $>0$, a contradiction. This shows that every point $z_{0} \in \mathrm{w}-\mathrm{cl}(\mathscr{Q})$ with $\lambda^{*} z_{0}=j$ (if any) is $\Lambda$-Pareto optimal for $\mathcal{Q}$.

REMARK 3.1. In the above, $Z$ was not required to be reflexive.

REMARK 3.2. If $a \in-\Lambda^{*}$, that is, $a \lambda \geqslant 0$ for all $\lambda \in \Lambda$, and if $j^{\prime}=\inf \{z=$ $a z \mid z \in \mathbb{Q}\}$ then the above lemma (with $\lambda^{*}=-a$ ) shows that for a nonempty $\Lambda$-bounded subset $\mathcal{Q}$ of $Z$, any point $z_{0} \in \mathrm{w}$-cl(Q) with $a z_{0}=j^{\prime}$ is $\Lambda$-Pareto optimal for $\mathbb{Q}$ in $Z$ provided $-a \in$ int $\Lambda^{*}$.

REMARK 3.3. The conclusion of Lemma 3.1 may not be true if the condition $\lambda^{*} \in$ int $\Lambda^{*}$ does not hold, as the following example shows. Take $Z=E^{2}$, $\Lambda=\left\{\lambda=\left(\lambda^{1}, \lambda^{2}\right) \mid \lambda^{1} \geqslant 0, \lambda^{2} \geqslant 0\right\}, \mathbb{Q}=\Lambda$, and thus $\Lambda^{*}=\left\{\left(\eta^{1}, \eta^{2}\right) \mid \eta^{1}<0\right.$, $\left.\eta^{2}<0\right\}$. The point $z=(0,0)$ is the only Pareto optimal point of $Q$ with respect to $\Lambda$. For $\eta=(0,-1)$, we have $\eta z=(0,-1)\left(z^{1}, z^{2}\right)=-z^{2}<0$ for all $z \in \mathbb{Q}$, and $j=0$. All points $z=\left(z^{1}, 0\right), z^{1} \geqslant 0$, belong to $\mathbb{Q}$ and satisfy $\eta z=0$, yet only $(0,0)$ is Pareto optimal.

4. Special cones and existence of optima. We introduce two special properties for cones in a Banach space that will be used later.

Definition 4.1. Given a Banach space $Z$, a closed convex cone $\Lambda$ and its polar $\Lambda^{*}$, we say that $\Lambda$ has property $(\pi)$ if there is at least one element $\lambda^{*} \in \Lambda^{*}$ such that for any $\delta>0$, the set $V\left(\lambda^{*}, \delta\right)=\left\{z \in \Lambda \mid \lambda^{*} z>-\delta\right\}$, if nonempty, is relatively weakly compact in $Z$.

REMARK 4.1. $\Lambda$ has property $(\pi)$ iff there is at least one element $a \in-\Lambda^{*}$ such that for any $\delta>0$, the set $V(a, \delta)=\{z \in \Lambda \mid a z<\delta\}$, if nonempty, is relatively weakly compact in $Z$. This property is of the type "weakly-infcompactness" of Moreau (see [23]).

REMARK. 4.2. If $Z$ is reflexive, the requirement $(\pi)$ is satisfied if for the linear functional $\lambda^{*} z$, that is, $\lambda^{*}: \Lambda \rightarrow E^{1}$, the reals, the inverse image of any 
bounded set is bounded; in other words $\left\{z \mid \lambda^{*} z \in B\right\}$ is bounded whenever $B$ is a bounded subset of the real line.

Another property of interest is given by

Definition 4.2. A closed convex cone $\Lambda$ in a Banach space $Z$ is said to have the angle property, if for some $\varepsilon$ and $a$, with $0<\varepsilon<1$ and $a \in-\Lambda^{*}$, $a \neq 0$, we have $\Lambda \subset\{z \in Z \mid a z>\varepsilon\|a\|\|z\|\}$.

REMARK 4.3. If $a \in Z^{*}$ is as in the angle property of a closed convex cone $\Lambda$, then $-a \in$ int $\Lambda^{*}$. Indeed, if $\lambda^{*} \in Z^{*}$ is with $\left\|\lambda^{*}+a\right\|<\varepsilon\|a\|$ (with $\varepsilon$ as in the angle property) then

$$
\left|\lambda^{*} z+a z\right| \leqslant\left\|\lambda^{*}+a\right\|\|z\|<\varepsilon\|a\|\|z\| \text {. }
$$

Thus, $\lambda^{*} z<-a z+\varepsilon\|a\|\|z\|<0$ (by the choice of $a$ ) for all $z \in \Lambda$. Thus, $\left\{\lambda^{*} \mid\left\|\lambda^{*}-(-a)\right\|<\varepsilon\|a\|\right\} \subset \Lambda^{*}$ or $-a \in$ int $\Lambda^{*}$.

REMARK 4.4. It is of interest to note that in any Banach space $Z$, a closed convex cone with angle property is clearly acute (being contained in $\{z|a z\rangle$ $0\} \cup\{0\}$, with $a$ from the angle property) and hence pointed. Also, a closed convex cone $\Lambda$ with property $(\pi)$ is acute (and hence pointed). Indeed, if $\Lambda$ is not acute, then for each $a \in Z^{*}$, there is a nonzero element $x_{a} \neq 0, x_{a} \in \Lambda$ with $a \cdot x_{a}<0$. Thus, for $\delta>0$ and for any fixed $a \in Z^{*}, a \neq 0$, the set $V_{a}=\{z \in \Lambda \mid a z \geqslant-\delta\}$ contains the unbounded set $\left\{\alpha x_{-a} \mid \alpha \geqslant 0, \alpha\right.$ real $\}$ where $x_{-a}$ is the nonzero element of $\Lambda$ with $-a x_{-a} \leqslant 0$ or $a x_{-a}>0$. Thus, $V_{a}$ is not weakly compact for any $a \in Z^{*}, a \neq 0$ and property $(\pi)$ is violated.

REMARK 4.5. If $Z$ is reflexive, it is easily seen that angle property implies property $(\pi)$. However, even in the Hilbert space

$$
l_{2}=\left\{\left(\alpha_{1}, \alpha_{2}, \ldots\right) \mid \alpha_{i} \text { real, } \sum_{i=1}^{\infty} \alpha_{i}^{2}<\infty\right\}
$$

we can find acute cones which do not have property $(\pi)$ nor the angle property. For example, let $\Lambda=\left\{\lambda \in l_{2} \mid \lambda_{i} \geqslant 0, i=1,2, \ldots\right\}$. Then, for any $a=\left(a_{1}, a_{2}, \ldots\right) \in \Lambda^{*}$, and any integer $n>0$, there is an index $i(n)$ with $a_{i(n)}>-n^{-1}$ and thus $\lambda^{n}=(0, \ldots, n, 0, \ldots)$ (with $n$ in the $i(n)$ th place, that is, $\lambda_{i(n)}^{n}=n$ and $\lambda_{i}^{n}=0$ for $i \neq i(n)$ ) belongs to $\{z \in \Lambda \mid a z \geqslant-1\}$ and this set is therefore not weakly compact. (It is to be noted that $\left\{\lambda^{n}\right\}$ is not bounded in norm.)

Also, $\Lambda$ does not have angle property because, for any $a \in-\Lambda^{*}, a=$ $\left(a_{1}, a_{2}, \ldots\right)$ and any $\varepsilon>0, \Lambda \subset\{z \mid a z \geqslant \varepsilon\|a\|\|z\|\}$ implies $a e^{i} \geqslant \varepsilon\|a\|\left\|e^{i}\right\|$ for any $i$ where $e^{i}=(0, \ldots, 1,0, \ldots)$, that is, $a_{i} \geqslant \varepsilon\|a\|$ for every $i$. Since $\varepsilon\|a\|$ is fixed, $a \neq 0$, and $a_{i} \rightarrow 0$ as $i \rightarrow \infty$ for $\left(a_{1}, a_{2}, \ldots\right) \in l_{2}$, we obtain a contradiction. However, $\Lambda$ is acute since $\Lambda \subset\left\{z \in l_{2} \mid a z>0\right\} \cup\{0\}$, where $a=\left(1,2^{-1}, \ldots\right)$, that is, $a_{i}=i^{-1}$. Indeed, $\lambda \in \Lambda$ implies $\lambda_{i} \geqslant 0$ for every $i$ and, thus, $\sum_{i=1}^{\infty}\left(i^{-1} \lambda_{i}\right) \geqslant 0$, while $\sum_{i=1}^{\infty}\left(i^{-1} \lambda_{i}\right)=0$ implies $\lambda_{i}=0$ for every $i$.

REMARK 4.6. If $Z$ is finite dimensional, then any closed convex cone which 
is acute has the angle property and, hence, also property $(\pi)$ and is pointed. Indeed, if $\Lambda$ is an acute closed convex cone, then $\Lambda \subset\{z \in Z \mid a z>0\} \cup$ $\{0\}$. Let $i=\inf \{a z \mid z \in \Lambda,\|z\|=1\}$. Then $i \geqslant 0$. Let $z_{n} \in \Lambda$, be chosen with $\left\|z_{n}\right\|=1$ and $a z_{n} \rightarrow i$ as $n \rightarrow \infty$. The sequence, being in a closed bounded subset of a finite dimensional space $Z$, has a (weakly) convergent subsequence, say still $z_{n}$, converging to an element $\bar{z} \in Z$. But $\bar{z} \in \Lambda$ since $\Lambda$ is closed. Thus, $a \bar{z}>0$ and $\|\bar{z}\|=1$ so that $a \bar{z}>0$. But $a \bar{z}=\lim a z_{n}=i$. Thus, $i>0$. For any $z \in \Lambda, z \neq 0$, we have $w=z /\|z\| \in \Lambda$ and $\|w\|=1$. Thus, by definition of $i$, we have $a w>a \bar{z}$, that is, $a z>a \bar{z}\|z\|=\varepsilon\|a\|\|z\|$, where $\varepsilon=a \bar{z} /\|a\|$. But then $\varepsilon>0$ since $a \bar{z}=i>0$, and

$$
\varepsilon=a \bar{z} /\|a\| \leqslant\|a\|\|\bar{z}\| /\|a\|=\|\bar{z}\|=1 \text {. }
$$

Thus, with this choice of $\varepsilon$ and $a, \Lambda \subset\{z \mid a z>\varepsilon\|a\|\|z\|\}$ and $\Lambda$ has the angle property.

EXAMPLe 4.1. To show that there exist "infinite dimensional" cones with the angle property, we consider the Hilbert space

$$
l_{2}=\left\{z \mid z=\left(z_{1}, z_{2}, \ldots\right), \sum_{i=1}^{\infty} z_{i}^{2}<\infty\right\} .
$$

Let $0<\alpha<1$ and $a^{\alpha}=\left(\alpha, \alpha^{2}, \ldots\right) \in l_{2}$ with $\left\|a^{\alpha}\right\|^{2}=\alpha^{2} /\left(1-\alpha^{2}\right)$. Then, for any $\varepsilon, 0<\varepsilon<\left(1-\alpha^{2}\right)^{1 / 2}$, the set $V\left(a^{\alpha}, \varepsilon\right)=\left\{z \in l_{2} \mid a^{\alpha} z>\varepsilon\left\|a^{\alpha}\right\|\|z\|\right\}$ contains elements $a^{\beta}=\left(\beta, \beta^{2}, \ldots\right) \in l_{2}$ with suitable $\beta$ in $0<\beta<1$. Indeed, if $\gamma_{1}, \gamma_{2}$ are the two distinct roots of the quadratic

$$
\beta^{2}\left(1-\alpha^{2}+\alpha^{2} \varepsilon^{2}\right)-2 \alpha \varepsilon^{2} \beta+\left(\varepsilon^{2}-1+\alpha^{2}\right)=0,
$$

then for $\gamma_{1}<\beta<\gamma_{2}$, the geometric sequence $a^{\beta}=\left(\beta, \beta^{2}, \ldots\right)$ belongs to $V\left(a^{\alpha}, \varepsilon\right)$ and the set $\left\{a^{\beta} \mid \gamma_{1}<\beta<\gamma_{2}\right\}$ cannot be imbedded in a finite dimensional subspace of $l_{2}$.

EXAMPLE 4.2. In any reflexive Banach space $Z$, if $z^{1}, \ldots, z^{n}$ are any $n$ fixed nonzero elements and $\bar{z}^{-1}, \ldots, \bar{z}^{n}$ are elements of $Z^{*}$ with $\bar{z}^{i}\left(z^{j}\right)=\delta_{i}$ $(=0$ for $i \neq j,=1$ for $i=j)$, then the set

$$
V\left(z^{1}, \ldots, z^{n}\right)=\left\{\lambda \in Z \mid \lambda=\sum_{i=1}^{n} \alpha_{i} z^{i}, \alpha_{i}>0, \alpha_{i} \text { real }\right\}
$$

is acute and has the angle property with $a=\sum_{i=1}^{n} \bar{z}_{i}$.

The above remarks and examples indicate the following diagram of implications.

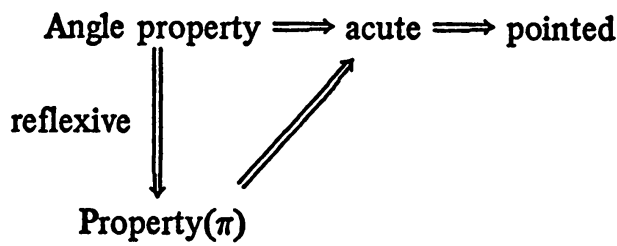


In finite dimensional spaces, all four properties are equivalent.

REMARK 4.7. Since angle property (as well as property $(\pi)$ ) implies acuteness, neither is satisfied for a halfspace. We illustrate this for $Z=E^{2}$, the 2-dimensional plane. Let $\Lambda=\{(x, y) \mid x>0\}$. Then for any $(\lambda, \mu) \neq$ $(0,0)$, and any $\delta>0$ real, for which the set $U_{\delta}=\{(x, y) \mid x>0, \lambda x+\mu y>$ $-\delta\}$ is nonempty, we have $U_{\delta}$ unbounded and, hence, not compact.

We shall now use the two properties introduced in this section to obtain the existence of $\Lambda$-optima for a $\Lambda$-bounded subset $\mathbb{Q}$.

LEMMA 4.1. Let $Z$ be any Banach space and let $\Lambda$ be a closed convex cone in $Z$, satisfying property $(\pi)$. For any $\Lambda$-bounded nonempty subset $\mathbb{Q}$ of $Z$, there exists at least one weak $\Lambda$-extremum point $\bar{z}$ in the weak closure of $\mathcal{Q}$. That is, $\mathbb{Q} \neq \varnothing, \mathbb{Q} \subset c+\Lambda$ for some $c \in Z$ implies $\mathrm{w}-\operatorname{Ext}_{\Lambda}(\mathbb{Q}) \neq \varnothing$. By definition, $\mathrm{w}-\operatorname{Ext}_{\Lambda}(\mathbb{Q}) \subset \mathrm{w}-\mathrm{cl}(\mathbb{Q})$ and thus, in particular, if $\mathbb{Q}$ is weakly closed in $Z$, then $w-\operatorname{Ext}_{\Lambda}(\mathbb{Q}) \subset \mathbb{Q}$.

Proof. Since $\Lambda$ has property $(\pi)$, it is also acute and thus there is an element $a \in Z^{*}$ with $a z>0$ for $z \in \Lambda-\{0\}$. Let $\mu^{*}=\lambda^{*}-a$ where $\lambda^{*} \in$ $\Lambda^{*}$ is given by property $(\pi)$, that is, for every $\delta>0$, the set $\left\{z \in \Lambda \mid \lambda^{*} z>\right.$ $-\delta\}$, if nonempty, is weakly relatively compact.

Since $\mathbb{Q}$ is $\Lambda$-bounded, there is $c \in Z$ with $\mathscr{Q} \subset c+\Lambda$ or $c<_{\Lambda} z$ for all $z \in \mathbb{Q}$. Since $\mu^{*} \in \Lambda^{*}, \mu^{*} z<\mu^{*} c$ and thus the set $\mu^{*} \mathbb{Q}=\left\{\mu^{*} z \mid z \in \mathbb{Q}\right\}$ is nonempty and bounded above. Let $j=\sup \mu^{*} \mathbb{Q}$. Then $j$ is finite. Let $z_{k} \in \mathbb{Q}$ be chosen so that $j-k^{-1} \leqslant \mu^{*} z_{k} \leqslant j$. Then

$$
0>\mu^{*}\left(z_{k_{c}}-c\right) \geqslant j-k^{-1}-\mu^{*} c>j-1-\mu^{*} c .
$$

Thus, $\lambda^{*}\left(z_{k}-c\right) \geqslant\left(\lambda^{*}-a\right)\left(z_{k}-c\right) \geqslant-\delta$ where $\delta=-\left(j-1-\mu^{*} c\right)>$ 0 . Using property $(\pi)$ and relabeling if necessary, we obtain $z_{0} \in Z$ with $z_{k} \rightarrow z_{0}$ weakly. Hence, $\mu^{*} z_{k} \rightarrow \mu^{*} z_{0}$ and $\mu^{*} z_{0}=j$. We claim that $z_{0} \in$ w-Ext($(\mathbb{Q})$. Indeed, $z_{0} \in \mathrm{w}-\mathrm{cl}(\mathbb{Q})$, and if there is $z \in \mathbb{Q}$ with $z \neq z_{0}$, and $z_{0}-z \in \Lambda$, then $\mu^{*} z_{0}<\mu^{*} z$, and since $\mu^{*} z_{0}=j$, we get $\mu^{*} z_{0}=\mu^{*} z$. But then $\lambda^{*}\left(z_{0}-z\right)=a\left(z_{0}-z\right)$, which is a contradiction unless $z_{0}=z$, because $\lambda^{*} \in \Lambda^{*}$ and $a \lambda>0$ for $\lambda \in \Lambda-\{0\}$. It follows that $z_{0} \in \mathrm{w}-\operatorname{Ext}(\mathbb{Q})$.

Remark 4.8. If $Z$ is reflexive, then by previous remarks, we may take $\Lambda$ to be a closed convex cone with angle property since then it has property $(\pi)$ also, and if $a$ is the element from angle property then $-a \in$ int $\Lambda^{*}$. However, with the angle property, we can obtain an estimate on $\|\bar{z}\|$. Indeed, if $\varepsilon$ and $a$ are given by angle property, $j-k^{-1}-\lambda^{*} c<\lambda^{*}\left(z_{k}-c\right)$ implies

$$
-j+k^{-1}+\lambda^{*} c>-\lambda^{*}\left(z_{k}-c\right)=a\left(z_{k}-c\right)>\varepsilon\|a\|\left\|z_{k}-c\right\|
$$

(since $-a=\lambda^{*} \in \Lambda^{*}$ satisfies the weak compactness condition in property ( $\pi)$ ). Thus,

$$
\left\|z_{k}\right\| \leqslant\|c\|+(\varepsilon\|a\|)^{-1}\left(-a c-j-k^{-1}\right)
$$


and

$$
\|\bar{z}\| \leqslant\|c\|+(\varepsilon\|a\|)^{-1}(-a c-j) .
$$

It is to be noted that $j\left\langle\lambda^{*} c=-a c\right.$ and, hence, $\left.-a c-j\right\rangle 0$.

REMARK 4.9. If $Z$ is reflexive, we may avoid property $(\pi)$ (as well as angle property) in the above lemma, provided (i) $z \in Q, z \neq 0$ implies $z /\|z\| \in \mathbb{Q}$, (ii) $\mathbb{Q} \subset c+\Lambda$ with $c \in \Lambda$, and (iii) int $\Lambda^{*} \neq \varnothing$. Indeed, in this case, we let $\lambda^{*}$ be any element of int $\Lambda^{*}$ and $j=\sup \left\{\lambda^{*} z \mid z \in \mathbb{Q}\right\}$. As before, this is finite and we get $z_{k} \in \mathbb{Q}$ with $j-k^{-1}<\lambda^{*} z_{k}<j$. We may construct a new sequence $\left\{w_{k}\right\} \subset \mathbb{Q}$ as follows: $w_{k}=z_{k}$ if $\left\|z_{k}\right\|<1$ and $w_{k}=z_{k} /\left\|z_{k}\right\|$ if $\left\|z_{k}\right\|>1$. Then $\lambda^{*} w_{k} \rightarrow j$ and $\left\|w_{k}\right\|<1$. By weak compactness of spheres in $Z$, there is a subsequence, say still $[k]$, and an element $\bar{w} \in \mathrm{w}-\mathrm{cl}(\mathbb{Q})$ with $w_{k} \rightarrow \bar{w}$ weakly as $k \rightarrow \infty$. Hence $\lambda^{*} w_{k} \rightarrow \lambda^{*} \bar{w}=j$ and the rest of the proof follows from Lemma 3.1.

The two conditions on $\mathcal{Q}$ are used to obtain $j-k^{-1}<\lambda^{*} w_{k}<j$. By (i), $w_{k} \in \mathbb{Q}$ and, hence, $\lambda^{*} w_{k}<j$. On the other hand, by (ii), $\mathbb{Q} \subset \Lambda$ and, hence, $\lambda^{*} z<0$ for all $z \in \mathbb{Q}$; hence, $j<0$ and $j-k^{-1}<0$ so that $\left\|z_{k}\right\|>1$ and $\left\|z_{k}\right\|^{-1}<1$ imply

$$
j-k^{-1}<\left(j-k^{-1}\right)\left\|z_{k}\right\|^{-1}<\lambda^{*} z_{k}\left(\left\|z_{k}\right\|\right)^{-1}=\lambda^{*} w_{k} .
$$

REMARK 4.10. Note that, by the argument of the proof of Lemma 4.1, we have shown the existence of at least one Pareto optimal point $\bar{z}=\bar{z}\left(\lambda^{*}\right)$ for every $\lambda^{*}$ satisfying property $(\pi)$. In Remark 4.11 we show by examples that this point $\bar{z}\left(\lambda^{*}\right)$ may not be unique for any given $\lambda^{*}$ satisfying $(\pi)$, and that $\bar{z}\left(\lambda^{*}\right)$ may not exist for $\lambda^{*}$ not satisfying property $(\pi)$. Finally, the set of all possible points $\bar{z}\left(\lambda^{*}\right)$ may be smaller than the set of the Pareto optimal points of $Q$.

REMARK 4.11. For $Z=E^{\rho}, 2 \leqslant \rho<\infty$, and

$$
\Lambda=\left[\lambda=\left(\lambda^{1}, \ldots, \lambda^{\rho}\right) \mid \lambda^{i} \geqslant 0, i=1, \ldots, \rho\right]=E_{+}^{\rho},
$$

property $(\pi)$ holds for every $\lambda^{*}=\eta=\left(\eta^{1}, \ldots, \eta^{\rho}\right)$, with $\eta^{i}<0, i=$ $1, \ldots, \rho$, as proved in Remark 4.6. For $Z=E^{\rho}$ and $\Lambda=E_{+}^{\rho}$, Lemma 4.1 proves that, for every $\eta=\left(\eta^{1}, \ldots, \eta^{\rho}\right), \eta^{i}<0, i=1, \ldots, \rho$, there exists at least a point $\bar{z}=\bar{z}(\eta) \in \operatorname{cl} \mathcal{Q}, \bar{z}(\eta) \in \operatorname{Ext}_{\Lambda} Q$, that is, $\bar{z}(\eta)$ is a Pareto optimal point of $\mathcal{Q}$. This point $\bar{z}(\eta)$ is not necessarily unique. For instance, if $Q=[(x, y) \mid x \geqslant 0, y \geqslant 0, x+y \geqslant 1]$, any point $(\alpha, 1-\alpha), 0<\alpha<1$, is Pareto optimal, and also a point $\bar{z}(\eta)$ for $\eta=(-1,-1)$.

Again, for $Z=E^{\rho}$ and $\Lambda=E_{+}^{\rho}, \rho>2$, the argument of the lemma does not apply for $\lambda^{*}=\eta=\left(\eta^{1}, \ldots, \eta^{\rho}\right)$ with $\eta^{i}<0$ for all $i$, and $\eta^{i}=0$ for at least one $i$, since then $U$ is not compact. Actually, for any such $\eta$, the point $\bar{z}(\eta)$ may not exist. For instance, if $\mathcal{Q}=[(x, y) \mid x>0, y>0, x y \geqslant 1]$, all points $(x, y)$ with $x \geqslant 0, y \geqslant 0, x y=1$ are Pareto optimal, but there are no 
points $\bar{z}(\eta)$ for $\eta=(0,1)$ and $\eta=(1,0)$, while $\bar{z}(\eta)$ is uniquely determined for $\eta=\left(\eta^{1}, \eta^{2}\right)$, with $\eta^{1}<0, \eta^{2}<0$, and these points $\bar{z}(\eta)$ cover the set of Pareto optimal points.

For $\mathfrak{Q}=\left[(x, y) \mid y \geqslant 0\right.$ if $1 \leqslant x<\infty ; y \geqslant 1-\left[1-(1-x)^{2}\right]^{1 / 2}$ if $0 \leqslant x$ $<1]$, the points $(x, y)$ with $y=1-\left[1-(1-x)^{2}\right]^{1 / 2}, 0<x<1$, are Pareto optimal, a point $\bar{z}(\eta)$ exists and is unique for every $\eta=\left(\eta^{1}, \eta^{2}\right) \neq 0, \eta^{1}<0$, $\eta^{2}<0$, and these are all the Pareto optimal points. On the other hand, for $\mathcal{Q}=\left[(x, y) \mid x \geqslant 0, y>0, x^{2}+y^{2} \geqslant 1\right]$, the points $(x, y)$ with $x>0, y>0$, $x^{2}+y^{2}=1$ are the Pareto optimal points, $\bar{z}(\eta)$ exists and is unique for every $\eta=\left(\eta^{1}, \eta^{2}\right) \neq 0, \eta^{1} \geqslant 0, \eta^{2} \geqslant 0$, yet $\bar{z}(\eta)$ can be either $(1,0)$ or $(0,1)$.

Remark 4.12. A detailed study of the geometry of Pareto extrema may be found in [2].

5. Notations. We deal here with a topological space $(X, \tau)$, with a Banach space $B$ with norm \|\| , and with metric spaces $(G, \rho),(Y, d),\left(U, d^{\prime}\right)$. In most applications $X$ will also be a Banach space, $\tau$ its weak topology; $B, Y$ Euclidean spaces $E^{r}, E^{s} ; G, U$ subsets of Euclidean spaces $E^{r}, E^{s}$. We assume that $G$ is also a finite, complete measure space $(G, \alpha, \mu)$ and $\mu$ is regular. Concerning $Y$ and $U$, we assume that they are $\sigma$-compact, that is, the countable union of compact subsets. Let $A$ be any subset in $G \times Y$ such that, for any $t \in G$, the set $A(t)=[y \in Y,(t, y) \in A]$ is nonempty. For every $(t, y) \in A$, let $U(t, y)$ be a given nonempty subset of $U$. Let $f(t, y, u)$ be a given function defined on the set

$$
S=[(t, y, u) \in G \times Y \times U \mid(t, y) \in A, u \in U(t, y)]
$$

with values in $B$, or $f: S \rightarrow B$. We assume that $A, S$ and the function $f$ satisfy a Caratheodory condition (C) on $G$, that is, given $\varepsilon>0$, there is a compact subset $K \subset G$ such that $\mu(G-K)<\varepsilon$, the sets $A_{K}=[(t, y) \in A, t \in K]$, $S_{K}=[(t, y, u) \mid t \in K]$ are closed in the product topologies of $(G, \rho) \times(Y, d)$ and $(G, \rho) \times(Y, d) \times\left(U, d^{\prime}\right)$, respectively, and $f: S \rightarrow B$, restricted to $S_{K}$ is continuous (in the weak topology of $B$ ). For any Hausdorff space $H$, we denote by $\mu(G, H)$ the set of all $\mu$-measurable functions on $G$ with values in $H$. For any Banach space $B$, we denote by $L_{1}(G, B)$ the set of all strongly Bochner integrable $\mu$-measurable functions $z$ on $G$ with values in $B$. For any $p, 1<p<\infty$, we denote by $L_{p}(G, B)$ the set of all $z \in L_{1}(G, B)$ with $\|z(\cdot)\| \in L_{p}\left(G, E^{1}\right)$ and then $\|z\|_{p}$ has the usual definition. Usual conventions hold for $p=\infty$. Thus, $L_{\infty}(G, B) \subset L_{p}(G, B) \subset L_{1}(G, B) \subset$ $\mu(G, B)$.

Let $L, M$ be two operators (not necessarily linear) with domains $D(L)$, $D(M) \subset X$ and values in $L_{1}(G, B), \mu(G, Y)$, respectively, and let $X_{0} \subset D(L)$ $\cap D(M)$ be a given nonempty part of their intersection. We shall consider the restrictions of $L$ and $M$ on $X_{0}$, or $L: X_{0} \rightarrow L_{1}(G, B), M: X_{0} \rightarrow \mu(G, Y)$. 
We shall say that a pair $x, u$ is admissible provided $x \in X_{0}, u \in \mu(G, U)$, and

$$
\begin{array}{r}
(M x)(t) \in A(t), \quad u(t) \in U(t,(M x)(t)), \quad(L x)(t)=f(t,(M x)(t), u(t)), \\
\mu \text {-a.e. in } G .
\end{array}
$$

Then we say that $y=M x \in \mu(G, Y)$ is a state function, and that $u \in$ $\mu(G, U)$ is a strategy, or control function.

We shall consider a given collection $\Omega$ of admissible pairs $x, u$. Then, we shall denote by $\{x\}_{\Omega}$ the collection $\{x\}_{\Omega}=\left\{x \in X_{0},(x, u) \in \Omega\right.$ for some $\left.u\right\}$, and we have $\{x\}_{\Omega} \subset X_{0} \subset X$.

Let $(X, \tau)$ be any topological space, $Z$ a Banach space, $X_{0}$ a subset of $X$, and $A: X_{0} \rightarrow Z$ a given operator, not necessarily linear.

The operator $A$ is said to have the strong (weak) closure property on $X_{0}$ with respect to $(X, \tau)$ provided $x_{k} \in X_{0}, k=1,2, \ldots, x_{k} \rightarrow x$ in $(X, \tau)$ as $k \rightarrow \infty, x \in X_{0}, A x_{k} \rightarrow y$ strongly (weakly) in $Z$ implies $A x=y$.

The operator $A$ is said to have the strong (weak) closed graph property on $X_{0}$ with respect to $(X, \tau)$ provided $x_{k} \in X_{0}, k=1,2, \ldots, x_{k} \rightarrow x$ in $(X, \tau)$ as $k \rightarrow \infty, x \in X, A x_{k} \rightarrow y$ strongly (weakly) in $Z$ implies that $x \in X_{0}$ and $A x=y$.

Whenever $X_{0}=X$, closure property and closed graph property coincide.

The operator $A$ is said to have the strong (weak) convergence property on $X_{0}$ with respect to $(X, \tau)$ provided $x_{k} \in X_{0}, k=1,2, \ldots, x_{k} \rightarrow x$ in $(X, \tau)$ as $k \rightarrow \infty$, implies that there is a subsequence $\left[k_{s}\right]$ such that $A x_{k_{s}}, s=1,2, \ldots$, is strongly (weakly) convergent in $Z$.

We define convergence in measure property similarly.

6. Existence theorems for Pareto-Mayer problems. For $(t, y) \in A$, we shall consider the sets

$$
Q(t, y)=f(t, y, U(t, y))=\{z \in B \mid z=f(t, y, u) ; u \in U(t, y)\} \subset B .
$$

We shall say that, for any given $t_{0} \in G$, the sets $Q(t, y)$ satisfy property $(Q)$ with respect to $y$ at the point $\left(t_{0}, y_{0}\right) \in A$ provided

$$
Q\left(t_{0}, y_{0}\right)=\bigcap_{\varepsilon>0} \operatorname{clco} \cup Q\left(t_{0}, y\right),
$$

where the union is taken for all $y \in A\left(t_{0}\right)$ in a neighborhood of radius $\varepsilon$ of $y_{0}$ (in the metric $d$ of $(Y, d)$ ). The sets $Q$ are said to have property (Q) with respect to $y$ in $A\left(t_{0}\right)$ if they have the above property at every $y_{0} \in A\left(t_{0}\right)$.

Finally, we assume that a functional $I[x]$ is defined in $\{x\}_{\Omega}$ with values in a Banach space $Z$, that is, $I:\{x\}_{\Omega} \rightarrow Z$. Let $\Lambda$ be a closed convex cone in $Z$ and $\Lambda^{*}$ be the polar cone of $Z$ in the dual space of $Z^{*}$. We say that the class $\Omega$ is closed (with respect to the Mayer problem under consideration) provided, whenever $\left(x_{k}, u_{k}\right) \in \Omega$, that is, $x_{k} \in\{x\}_{\Omega}, k=1,2, \ldots, x_{k} \rightarrow x$ in $(X, z)$ as 
$k \rightarrow \infty, x \in X_{0}$, and there is some $u \in \mu(G, U)$ such that $x, u$ is admissible, then there is also some $\bar{u} \in \mu(G, U)$ with $(x, \bar{u}) \in \Omega$; thus, $x \in\{x\}_{\Omega}$.

The class $\Omega$ of all admissible pairs $(x, u)$ is certainly closed in the sense above. This particular form of our definition is justified by the closure theorem 5.4 of [19] with $B_{2}=\{0\}$.

THEOREM 6.1 (Existence theorem for Pareto-Mayer problems). Let $Z$ be any Banach space over the reals, $\Lambda$ a closed convex cone in $Z, \Lambda^{*}$ the polar of $\Lambda$, with int $\Lambda^{*} \neq \varnothing$. Let $A, S, f$ satisfy condition (C). Let us assume that $\Omega$ is nonempty and closed, that $\{x\}_{\Omega}$ is sequentially relatively compact in $(X, \tau)$, and that the functional $I:\{x\}_{\Omega} \rightarrow Z$ is $\Lambda$-bounded in $Z$. Also, for some $a \in Z^{*}$ with $-a \in$ int $\Lambda^{*}$ let aI: $\{x\}_{\Omega} \rightarrow E^{1}$ be lower semicontinuous (with respect to $\Omega$ ), that is, $x_{k} \rightarrow x$ in $(X, \tau)$ and $x \in\{x\}_{\Omega}$ implies $a I[x]<\lim _{\text {inf }} \operatorname{lom}_{k \rightarrow \infty} a I\left[x_{k}\right]$.

Let us assume that both the operators $L$ and $M$ have the closure property in $X_{0}$ and that at least one of them has the closed graph property in $X_{0}$. Finally, let us require that $(A Q): L: X_{0} \rightarrow L_{1}(G, B)$ has the weak convergence property relative to $\{x\}_{\Omega} ; M: X_{0} \rightarrow \mu(G, Y)$ has the convergence in measure property relative to $\{x\}_{\Omega}$; and that, for $\mu$-almost all $t \in G$, the subsets $Q(t, y)$ of $B$ are closed, convex and have property $(Q)$ with respect to $y$ in $A(t)$. Then the functional $I[x]$ has at least one $\Lambda$-Pareto minimum in $\{x\}_{\Omega}$. (Specifically, aI has a minimum.)

Mere property $(\mathrm{K})$ suffices, instead of property $(\mathrm{Q})$, if the space $B$ is finite dimensional (cf. Remark 7.3).

Proof. By hypothesis $I[x]>_{\Lambda} c$ for some element $c \in Z$ and all $x \in\{x\}_{\Omega}$, or $I[x] \subset c+\Lambda$. Thus, the set $\mathbb{Q}=\left\{z \in Z, z=I[x], x \in\{x\}_{\Omega}\right\}$ is nonempty and bounded below in $Z$ with respect to $\Lambda$. Let us choose an element $a$ with $\lambda^{*}=-a \in$ int $\Lambda^{*}$ and for which $a I$ is lower semicontinuous, and note that for this $\lambda^{*}$ the set of real numbers $\left\{z=\lambda^{*} z, z \in \mathbb{Q}\right\}$, or $\left\{z=\lambda^{*} I[x], x \in\{x\}_{\Omega}\right\}$, is bounded above and nonempty, so that its supremum $j$ is finite. Let $\left[z_{k}\right]$ be a sequence of points of $\mathbb{Q}$, or $z_{k}=I\left[x_{k}\right]$, $x_{k} \in\{x\}_{\Omega}, k=1,2, \ldots$, such that $\lambda^{*} z_{k} \rightarrow j$. By hypothesis, $\{x\}_{\Omega}$ is relatively weakly compact in $X$; hence, there is a subsequence, say still $[k]$ for the sake of simplicity, and an element $x \in X$ such that $x_{k} \rightarrow x$ in $(X, \tau)$.

Because of the assumed convergence properties of $L$ and $M$, there is a subsequence, say still [ $k$ ], such that $L x_{k} \rightarrow y$ weakly for some $y \in L_{1}(G, B)$, $M x_{k} \rightarrow z$ for some $z \in \mu(G, B)$, and thus, for a suitable further subsequence, say still [ $k$ ], we also have $M x_{k}(t) \rightarrow M x(t), \mu$-a.e. in $G$. Because of the closure properties of $L$ and $M$ we have $L x=y, M x=z$, and because of the closed graph property of at least one of the operators $L$ and $M$, we also have $x \in X_{0}$. By closure theorem 5.4 [19] there is some $\bar{u}(t), t \in G, \bar{u} \in$ $\mu(G, U)$-which may depend on $\lambda^{*}$-such that $(x, \bar{u})$ is admissible. By 
hypothesis, there is some element $u, u \in \mu(G, U)$-which again may depend on $\lambda^{*}$-such that $(x, u)$ is also admissible, $(x, u) \in \Omega, x \in\{x\}_{\Omega}$, and by lower semicontinuity of $a I$,

$$
a I[x]<\liminf _{k \rightarrow \infty} a I\left[x_{k}\right]=\lim _{k \rightarrow \infty} a z_{k}=-j,
$$

or $-a I[x] \geqslant j$. Thus, $\mathscr{Z}=\lambda^{*} z \geqslant j$ for $z=I[x]$. But since $x \in\{x\}_{\Omega}, I[x] \in$ $\mathscr{Q}$ and, thus, $\mathscr{Z}=\lambda^{*} z \leqslant j$. We conclude that $\mathscr{Z}=\lambda^{*} z=\lambda^{*} I[x]=j$. Finally, $z \in \mathbb{Q}, \lambda^{*} z=j, \lambda^{*} \in$ int $\Lambda^{*}$ and, by Lemma $3.1, z$ is $\Lambda$-Pareto optimal for $\mathcal{Q}$ in $Z$. In other words, $x \in\{x\}_{\Omega}$ is $\Lambda$-Pareto optimal for the $Z$-valued functional $I[x]$ in $\{x\}_{\Omega}$. Theorem 6.1 is thereby proved.

REMARK 6.1. By using variants of the closure theorem mentioned in the above proof, the same conclusions as in the above theorem can be drawn under a variety of alternate hypotheses, as in Cesari [5]. We mention three of these below, for illustration purposes, which are relevant if $B$ is not finite dimensional.

(a) [(AQ) replaced by $\left(\mathrm{AQ}^{*}\right)$ ]: Let $B=B_{1} \times B_{2}, B_{1}, B_{2}$ Banach, $Y, U$ metric, $L=L_{1} \times L_{2}, f=\left(f_{1}, f_{2}\right), f_{i}: S \rightarrow B_{i}, L_{i}: X_{0} \rightarrow L_{1}\left(G, B_{i}\right), i=1,2 ; M$ : $X_{0} \rightarrow \mu(G, Y)$ and equation $L x=f$ replaced by $L_{i}(x(t))=f_{i}(t, M x(t), u(t))$, $t \in G, i=1$, 2. We assume that $L_{1}$ has weak convergence property, $L_{2}$ has strong convergence property (all relative to $\{x\}_{\Omega}$ ). We assume finally that for every $t \in G-T_{0}$, the sets $\tilde{Q}(t, y)$ satisfy property $\left(\mathrm{Q}^{*}\right)$ with respect to $y$ in $A(t)$ relative to $\left(B_{1}, B_{2}\right)$. [For definition of property $\left(\mathrm{Q}^{*}\right)$ see Cesari [5], or Kaiser and Suryanarayana [19].] Here we assume that $L_{1}, L_{2}, M$ have closure property in $X_{0}$ and that at least one of them has the closed graph property in $X_{0}$.

(b) (AQ) replaced by (AP): $B$ is a Banach space with the following properties: $\left(\mathrm{R}_{1}\right)$ Any sequence $z_{k}(t), t \in G, z_{k} \in L_{\infty}(G, B), k=1,2, \ldots$, with $\left\|z_{k}\right\|_{\infty}<N$ for all $k$, possesses a subsequence which is weakly convergent in $L_{\infty}(G, B) .\left(\mathrm{R}_{2}\right)$ Any sequence $z_{k}(t), t \in G, z_{k} \in L_{1}(G, B), k=$ $1,2, \ldots$, which is weakly convergent in $L_{1}(G, B)$ is equiabsolutely integrable in $G$.

We assume $L: X_{0} \rightarrow L_{1}(G, B)$ has weak convergence property relative to $\{x\}_{\Omega}$ and $M: X_{0} \rightarrow \mu(G, Y)$ has convergence in measure property relative to $\{x\}_{\Omega}$. We also assume that the sets $Q(t, y)$ are closed and convex and satisfy the conditions: $\left(\mathrm{P}_{1}\right)$ there is a bounded $\mu$-measurable function $p(t), t \in G$, $p \in \mu(G, B), p: G \rightarrow B,\|p(t)\|_{B} \leqslant \sigma$ for all $t \in G$ such that $p(t) \in Q(t, y)$ for all $t \in G-T_{0}$ and $y \in A(t)$. ( $\left.\mathrm{P}_{2}\right)$ For every $t \in G-T_{0}$, and $N>\sigma$ (with $\sigma$ as in $\left(\mathrm{P}_{1}\right)$ ) the closed convex equibounded sets $Q(t, y) \cap V(0, N)$ satisfy property $(\mathrm{Q})$ with respect to $y$ in $A(t)$. Here $V(0, N)=\{z \in B \mid$ $\|z\|<N\}$.

(c) ((AQ) replaced by (Fp), $1<p<\infty)$. Here both $B$ and $Y$ are Banach 
spaces and $U$ is a metric space. Let $L: X_{0} \rightarrow L_{1}(G, B)$ have weak convergence property relative to $\{x\}_{\Omega}$. Also, let $M: X_{0} \rightarrow L_{p}(G, Y)$ have strong $L_{p^{-}}$ convergence property, relative to $\{x\}_{\Omega}$. The sets $U(t)$ depend only on $t$, the sets $Q(t, y)$ are closed and convex. For $1 \leqslant p \leqslant \infty$, there are constants $c, \gamma, \zeta_{0}, p^{\prime}$ with $c \geqslant 0, \zeta_{0} \geqslant 0,0<\gamma \leqslant p, p^{\prime}=p(p-\gamma)^{-1}$ if $0<\gamma<p$, $p^{\prime}=\infty$ if $\gamma=p$ and functions $F(t) \geqslant 0, t \in G, F \in L_{p^{\prime}}\left(G, E^{1}\right), h(\zeta), 0<\zeta$ $<\infty, h$ monotone nondecreasing, $h(0+)=0, h(\zeta) \leqslant c|\zeta|^{\gamma}$ for $\zeta \geqslant \zeta_{0}$ such that, for all $(t, y, u),\left(t, y_{2}, u\right) \in S, t \in G-T_{0}$, we have

$$
\left\|f\left(t, y_{1}, u\right)-f\left(t, y_{2}, u\right)\right\|_{B} \leqslant F(t) h\left(\left\|y_{1}-y_{2}\right\|_{Y}\right) \text {. }
$$

For $p=\infty$, this same relation holds for some $F(t) \geqslant 0, t \in G, F \in$ $L_{1}\left(G, E^{1}\right), h(\zeta), 0<\zeta<+\infty, h$ monotone nondecreasing with $h(0+)=0$.

REMARK 6.2. For some other variations, see also the remarks for Lagrange problems in the next section. See, in particular, Remark 7.3. The sufficiency of property $(\mathrm{K})$, instead of property $(\mathrm{Q})$, whenever the space $B$ is finite dimensional was proved by Cesari and Suryanarayana in [34].

7. A lower closure theorem. In the study of Mayer problems in the last section, we were able to apply known closure theorems to obtain the existence of Pareto optimal solutions, with respect to a general closed convex cone $\Lambda$ (with no requirement of angle property) in a general Banach space $Z$ (with no reflexivity condition). This is mainly due to the fact that the cost functional $I$ is assumed to be such that $a I$ is lower semicontinuous for some $a$ with $-a \in$ int $\Lambda^{*}$. This freedom is certainly curtailed when we consider Lagrange problems. We shall assume $Z$ to be reflexive and the closed convex cone $\Lambda$ to satisfy an angle property. We shall prove a relevant lower closure theorem in the same lines as Theorem (5.i) of [7], applicable to the Pareto optimal problems. For completeness, we incorporate the proof here.

THEOREM 7.1. Let $Z$ and $B$ be Banach spaces. Let $Z$ be reflexive and let $\Lambda$ be $a$ closed convex cone in $Z$, with the angle property. Thus, $\Lambda \subset\{x \in Z|a x\rangle$ $\varepsilon\|a\|\|x\|\}$ for some $a \in Z^{*}, a \neq 0$ and $0<\varepsilon \leqslant 1$. Let $(G, \rho),(Y, d)$ be metric spaces and $G$ be a complete measure space with a finite measure $\mu$ and $\sigma$-algebra $\alpha$ of $\mu$-measurable subsets. Let $A$ be any subset of $G \times Y$ such that, for $t \in G$, the set $A(t)=\{y \in Y \mid(t, y) \in A\}$ is nonempty. For each $(t, y) \in A$, let $\bar{Q}(t, y)$ be a nonempty subset of $Z \times B$.

Let $T_{0} \in \alpha, \mu\left(T_{0}\right)=0$ be such that for $t \in G-T_{0}, A(t)$ is closed and $\bar{Q}(t, y)$ is closed and convex and satisfies property (Q) with respect to $y \in$ $A(t)$. Let $\xi(t), y(t), \eta_{k}(t), \xi_{k}(t), y_{k}(t), \phi(t), t \in G, k=1,2, \ldots$, be $\mu-$ measurable functions, $\xi, \xi_{k} \in L_{1}(G, B), \eta_{k}, \phi \in L_{1}(G, Z)$ such that

(i) $y_{k}(t) \in A(t),\left(\eta_{k}(t), \xi_{k}(t)\right) \in \tilde{Q}\left(t, y_{k}(t)\right), t \in G($ a.e. $), k=1,2, \ldots$;

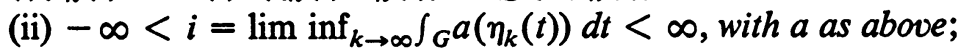

(iii) $\xi_{k} \rightarrow \xi$ weakly in $L_{1}(G, B), y_{k}(t) \rightarrow y(t)$ in measure in $G$ as $k \rightarrow \infty$; 
(iv) $\eta_{k}(t)-\phi(t) \in \Lambda, t \in G, \mu$-a.e.,

then, there is a function $\eta(t), t \in G, \eta \in L_{1}(G, Z)$ such that $y(t) \in A(t),(\eta(t)$, $\xi(t)) \in \tilde{Q}(t, y(t)), t \in G($ a.e. $)$, and $\int_{G} a(\eta(t)) d t<i$.

Mere property $(\mathrm{K})$ suffices, instead of property $(\mathrm{Q})$, if the space $B$ is finite dimensional (cf. Remark 7.3).

Proof. Let $j_{k}=\int_{G} a\left(\eta_{k}(t)\right) d t, k=1,2, \ldots$ By taking suitable subsequences we may assume that $j_{k} \rightarrow i$ and $y_{k}(t) \rightarrow y(t)$ a.e. in $G$ as $k \rightarrow \infty$. Here, $-\infty<i<\infty$, so that if $\rho_{s}=\max \left\{\left|j_{k}-i\right|, k \geqslant s+1\right\}$ then $\rho_{s} \rightarrow 0$ as $s \rightarrow \infty$. Let $T_{0}^{\prime} \in \alpha$ with $\mu\left(T_{0}^{\prime}\right)=0$ be such that $y_{k}(t) \rightarrow y(t)$ for all $t \in G-$ $T_{0}^{\prime}$. Then $y(t) \in A(t)$ for $t \in G-\left(T_{0} \cup T_{0}^{\prime}\right)$.

For $s=1,2, \ldots$, consider the sequence $\xi_{s+k}(t), t \in G$, which converges to $\xi(t)$ weakly in $L_{1}(G, B)$ as $k \rightarrow \infty$. By Mazur's theorem, there is a set of real numbers $c_{s N k} \geqslant 0, k=1, \ldots, N, N=1,2, \ldots$, with $\sum_{k=1}^{N} c_{s N k}=1$ and such that $\xi_{N}^{s}(t)$, defined as $\sum_{k=1}^{N} c_{s N k} \xi_{s+k}(t)$, converges strongly in $L_{1}(G, B)$ to $\xi(t)$. This is true for $s=1,2, \ldots$ For each $s$, there is a subset $T_{s} \in \alpha$, $\mu\left(T_{s}\right)=0$ and a subsequence $N_{h}^{s}, h=1,2, \ldots$, with $N_{h}^{s} \rightarrow \infty$ such that, for $t \in G-T_{s}, \xi_{N_{h}}^{s}(t) \rightarrow \xi(t)$ as $h \rightarrow \infty$.

Let $T_{0}^{\prime \prime}$ be the set of measure zero such that $\eta_{k}(t)-\phi(t) \in \Lambda$ for all $k$ and $t \in G-T_{0}^{\prime \prime}$. Defining

$$
\eta_{N}^{s}(t)=\sum_{k=1}^{N} c_{s N k} \eta_{s+k}(t)
$$

(just as $\xi_{N}^{s}$ ), we have

$$
\eta_{N}^{s}(t)-\phi(t) \in \Lambda \text { and } i-\rho_{s}<\int_{G} a\left(\eta_{N}^{s}(t)\right) d t<i+\rho_{s}
$$

For $N=N_{h}^{s}$ and $h \rightarrow \infty$, we obtain from Fatou's Lemma and the relation $a\left(\eta_{N}^{s}(t)-\phi(t)\right) \geqslant 0$ that the function $\theta^{s}(t)$ defined by

$$
\theta^{s}(t)=\liminf _{h \rightarrow \infty} a \cdot \eta_{N_{h}}^{s}(t)
$$

satisfies the relations

$$
\theta^{s}(t)>a(\phi(t)), \quad t \in G \text { a.e., }
$$

and

$$
\int_{G} \theta^{s}(t) d t<\liminf _{h \rightarrow \infty} \int_{G} a\left(\eta_{N_{h}}^{s}(t)\right) d t<i+\rho_{s}, \quad s=1,2, \ldots
$$

Thus, $\theta^{s}(t)$ is finite a.e. in $G$ and of class $L_{1}(G)=L_{1}\left(G, E^{1}\right)$. Let $T_{s}^{\prime}$ denote the set of measure zero of points $t \in G$ where $\theta^{s}(t)$ is not finite. Define

$$
\theta(t)=\liminf _{s \rightarrow \infty} \theta^{s}(t), \quad t \in G .
$$

Then, $\theta(t)>a(\phi(t))$, a.e. and $\int_{G} \theta(t) d t<i$. Thus, $\theta(t)$ is finite a.e. in $G$ and of class $L_{1}(G)$. Let $T_{0}^{\prime \prime \prime}$ be the set of measure zero where $\theta(t)$ is not finite, and 
let $T$ be the union of all sets $T_{0}, T_{0}^{\prime}, T_{0}^{\prime \prime}, T_{0}^{\prime \prime \prime}, T_{s}, T_{s}^{\prime}, s=1,2, \ldots$ Let $t_{0} \in G-T$, and let $s_{\omega}\left(t_{0}\right)$ be a subsequence such that $\theta\left(t_{0}\right)=\lim _{\omega \rightarrow \infty} \theta^{s \omega}\left(t_{0}\right)$ and $\left|\theta^{s \omega}\left(t_{0}\right)\right|<\left|\theta\left(t_{0}\right)\right|+1$.

For each fixed $s_{\omega}$, let $\left\{\eta_{\gamma}^{s \omega}\left(t_{0}\right)\right\}$ be a subsequence of $\left\{\eta_{N_{h}}^{s \omega}\left(t_{0}\right)\right\}$ such that

$$
\theta^{s \omega}\left(t_{0}\right)=\lim _{\gamma \rightarrow \infty} a \eta_{\gamma}^{s \omega}\left(t_{0}\right)
$$

and

$$
\left|a \eta_{\gamma}^{s \omega}\left(t_{0}\right)\right|<\left|\theta^{s \omega}\left(t_{0}\right)\right|+1<\left|\theta\left(t_{0}\right)\right|+2 .
$$

Thus,

$$
\left|a\left(\eta_{\gamma}^{s \omega}\left(t_{0}\right)-\phi\left(t_{0}\right)\right)\right|<\left|\theta\left(t_{0}\right)\right|+\left|a \phi\left(t_{0}\right)\right|+2 .
$$

But since $\eta_{\gamma}^{s \omega}\left(t_{0}\right)-\phi\left(t_{0}\right) \in \Lambda$, by choice of $a$ and $\varepsilon$, it follows that

$$
\left\|\eta_{\gamma}^{s \omega}\left(t_{0}\right)-\phi\left(t_{0}\right)\right\|_{Z}<\left[\left|\theta\left(t_{0}\right)\right|+\left|a\left(\phi\left(t_{0}\right)\right)\right|+2\right] \varepsilon^{-1}\|a\| .
$$

Thus, $\left\|\eta_{\gamma}^{s \omega}\left(t_{0}\right)\right\|$ is bounded and, by weak compactness of spheres in $Z$, there is a subsequence, say $\{\gamma\}$ still, and an element $\eta_{s \omega}\left(t_{0}\right)$ such that $\eta_{\gamma}^{s \omega}\left(t_{0}\right) \rightarrow$ $\eta_{s \omega}\left(t_{0}\right)$ weakly as $\gamma \rightarrow \infty$. Then, $\eta_{s \omega}\left(t_{0}\right)-\phi\left(t_{0}\right) \in \Lambda, a \eta_{s \omega}\left(t_{0}\right)=\theta^{s \omega}\left(t_{0}\right)$, and

$$
\left\|\eta_{s \omega}\left(t_{0}\right)\right\|_{z}<\left\|\phi\left(t_{0}\right)\right\|_{z}+\left[\left|\phi\left(t_{0}\right)\right|+\left|a\left(\phi\left(t_{0}\right)\right)\right|+2\right] \varepsilon^{-1}\|a\| .
$$

By repeating the above argument, with $\eta_{s \omega}\left(t_{0}\right)$, we observe that there is a subsequence, say $s \omega$ still, and an element $\eta\left(t_{0}\right)$, such that $\eta_{s \omega}\left(t_{0}\right) \rightarrow \eta\left(t_{0}\right)$ weakly in $Z$ as $\omega \rightarrow \infty$ and

$$
\left\|\eta\left(t_{0}\right)\right\|_{z} \leqslant\left\|\phi\left(t_{0}\right)\right\|_{z}+\left[\left|\theta\left(t_{0}\right)\right|+\left|a \phi\left(t_{0}\right)\right|+2\right] \varepsilon^{-1}\|a\| .
$$

Also, $a \eta\left(t_{0}\right)=\theta\left(t_{0}\right)$.

Setting $y_{0}=y\left(t_{0}\right)$, we have $\left(t_{0}, y_{0}\right) \in A$ and the sets $\tilde{Q}\left(t_{0}, y\right)$ have property (Q) at $y_{0}$. Given $\delta>0$ there is some $s_{0}$ such that $d\left(y_{s}\left(t_{0}\right), y_{0}\right)<\delta$ for $s>s_{0}$. For $s \geqslant s_{0}$, we have $\left(\eta_{s+k}\left(t_{0}\right), \xi_{s+k}\left(t_{0}\right)\right) \in \tilde{Q}\left(t_{0}, y_{s+k}\left(t_{0}\right)\right)$ and $d\left(y_{s+k}\left(t_{0}\right), y_{0}\right)$ $<\delta, k=1,2, \ldots$, and, hence,

$$
\left(\eta_{N}^{s}\left(t_{0}\right), \xi_{N}^{s}\left(t_{0}\right)\right) \in \operatorname{co} \bigcup\left\{\tilde{Q}\left(t_{0}, y\right) \mid d\left(y, y_{0}\right) \leqslant \delta\right\} .
$$

Finally, for $N=N_{h}^{s}$ and $h \rightarrow \infty,\left(\eta_{N}^{s}\left(t_{0}\right), \xi_{N}^{s}\left(t_{0}\right)\right)$ is a sequence with $\left(\eta\left(t_{0}\right)\right.$, $\left.\xi\left(t_{0}\right)\right)$ as an accumulation point. Thus,

$$
\left(\eta\left(t_{0}\right), \xi\left(t_{0}\right)\right) \in \operatorname{cl} \operatorname{co} \bigcup\left\{\widetilde{Q}\left(t_{0}, y\right) \mid d\left(y, y_{0}\right)<\delta\right\} .
$$

Since $\delta>0$ is arbitrary, by property $(\mathrm{Q})$, we have

$$
\left(\eta\left(t_{0}\right), \xi\left(t_{0}\right)\right) \in \bigcap_{\delta>0} \operatorname{cl} \operatorname{co} \bigcup\left\{\tilde{Q}\left(t_{0}, y\right) \mid d\left(y, y_{0}\right)<\delta\right\}=\tilde{Q}\left(t_{0}, y_{0}\right) .
$$

Thus, $(\eta(t), \xi(t)) \in \tilde{Q}(t, y(t))$ for all $t \in G-T$. Also,

$$
\|\eta(t)\|_{Z} \leqslant\|\phi(t)\|_{Z}+[|\phi(t)|+|a \phi(t)|+2] \varepsilon^{-1}\|a\|
$$


for $t \in G-T$ and the right-hand side of this inequality belongs to $L_{1}\left(G, E^{1}\right)$. Thus, $\eta(t)$ is Bochner integrable $\eta \in L_{1}(G, Z)$. Furthermore, $a \eta=\theta$ implies $\int_{G} a \eta(t) d t \leqslant i$. This completes the proof.

REMARK 7.1. Requirement (iv) in the above theorem can be replaced by the weaker assumption:

There exist $\phi_{k}, \phi \in L_{1}\left(G, E^{1}\right)$ such that $a \eta_{k}(t) \geqslant \phi_{k}(t), t \in G(\mu$-a.e.) (with $a$ as above).

The following implicit function theorem of McShane and Warfield [22] has been used in the past to obtain lower closure theorems for control problems as particular cases of those for orientor fields.

THEOREM 7.2. Let $G$ be a measure space, $H$ a Hausdorff space and $X$ a topological space which is $\sigma$-compact, that is, $X$ is a countable union of compact metrizable subsets. Let $g: X \rightarrow H$ be a continuous function and $\Phi: G \rightarrow H$ be a measurable function such that $\Phi(G) \subseteq g(X)$. Then there is a measurable function $\psi: G \rightarrow X$ such that $g(\psi(t))=\Phi(t), t \in G$.

As a consequence of Theorems 7.1 and 7.2, we obtain

THEOREM 7.3 (A lower closure theorem for Pareto-Mayer control problems). Let $G, Y, U, B, S, f$ and $A$ be as in $\S 5$. Let $Z$ be a reflexive Banach space and $\Lambda$ be a closed convex cone in $Z$ with the angle property so that for some $a \in-\Lambda^{*}$ and $0<\varepsilon \leqslant 1, \Lambda \subset\{z \mid a z \geqslant \varepsilon\|a\|\|z\|\}$. Let $g: S \rightarrow Z$ and let $f, g, A$ and $S$ satisfy Carathéodory condition (C). Let

$$
\tilde{Q}(t, y)=\left\{\left(z^{0}, z\right) \in Z \times B \mid z^{0}=g(t, y, u), z=f(t, y, u), u \in U(t, y)\right\}
$$

and

$$
\begin{aligned}
\tilde{Q}(t, y) & =\tilde{Q}(t, y)+\Lambda \\
& =\left\{\left(z^{0}, z\right) \in Z \times B \mid z^{0} \in g(t, y, u)+\Lambda, z=f(t, y, u), u \in U(t, y)\right\} .
\end{aligned}
$$

Let $T_{0} \in \alpha, \mu\left(T_{0}\right)=0$ be such that for $t \in G-T_{0}, A(t)$ is closed and $\tilde{Q}(t, y)$ is closed and convex and satisfies property $(\mathrm{Q})$ with respect to $y \in A(t)$. Let $\xi_{k}(t), \xi(t), y_{k}(t), y(t), u_{k}(t), \eta_{k}(t), \phi(t), t \in G, k=1,2, \ldots$, be measurable functions on $G$ such that

(i) $y_{k}(t) \in A(t), u_{k}(t) \in U\left(t, y_{k}(t)\right), \quad \xi_{k}(t)=f\left(t, y_{k}(t), \quad u_{k}(t)\right), \quad \eta_{k}(t)=$ $g\left(t, y_{k}(t), u_{k}(t)\right), \mu$-a.e. in $G, k=1,2, \ldots$;

(ii) $\xi_{k}, \xi \in L_{1}(G, B), \eta_{k}, \phi \in L_{1}(G, Z)$;

(iii) $-\infty<i=\lim _{\inf _{k \rightarrow \infty} \int} a \eta_{k}(t) d t<\infty$, with a as above;

(iv) $\xi_{k} \rightarrow \xi$ weakly in $L_{1}(G, B), y_{k}(t) \rightarrow y(t)$ in measure in $G$ as $k \rightarrow \infty$;

(v) $\eta_{k}(t)-\phi(t) \in \Lambda, t \in G$ a.e.;

then there are $\mu$-measurable functions $\bar{\eta}(t), u(t), t \in G$, such that $\bar{\eta} \in L_{1}(G, Z)$ and $y(t) \in A(t), u(t) \in U(t, y(t))$, and if $\eta(t)$ denotes $g(t, y(t), u(t))$, then 
$\bar{\eta}(t)-\eta(t) \in \Lambda, \quad \xi(t)=f(t, y(t), u(t)) \quad \mu$-a.e. in $G$, and $\int_{G} a \eta(t) d \mu<$ $\int_{G} a \bar{\eta}(t) d \mu<i$.

Mere property (K), instead of property (Q), suffices if the spaces $B$ and $S$ are finite dimensional (cf. Remark 7.3).

ReMARK 7.2. Without additional assumptions we may have $\int_{G} a \eta(t) d \mu=$ $-\infty$ and $\eta \notin L_{1}(G, Z)$. This happens even in the case $Z=E^{1}$ (see Examples 1, 2 of Cesari [7]). On the other hand, we can guarantee that $a \eta \in$ $L_{1}\left(G, E^{1}\right)$ and even that $\eta \in L_{1}(G, Z)$, if the following "lower bound" condition $(\alpha)$ holds.

$(\alpha)$ There is a real valued function $\phi_{a} \in L_{1}\left(G, E^{1}\right)$ such that $a g(t, y, u) \geqslant$ $\phi_{a}(t)$ for all $(t, y, u) \in S$.

Indeed, since $\bar{\eta}(t)-\eta(t) \in \Lambda$, we have

$$
a(\bar{\eta}(t)-\eta(t)) \geqslant \varepsilon\|a\|\|\bar{\eta}(t)-\eta(t)\|
$$

and

$$
\begin{aligned}
\|\eta(t)\| & \leqslant\|\bar{\eta}(t)\|+\varepsilon^{-1}\|a\|^{-1} a(\bar{\eta}(t)-\eta(t)) \\
& \leqslant\|\bar{\eta}(t)\|+\varepsilon^{-1}\left[a(\bar{\eta}(t))-\phi_{a}(t)\right] .
\end{aligned}
$$

Since the right-hand side of this inequality is in $L_{1}\left(G, E^{1}\right)$, it follows that $\eta$ is Bochner integrable.

For other similar lower bound conditions, we refer to Cesari [7].

The following lower closure theorem requires an analytic condition instead of property $(\mathrm{Q})$. The proof is in the same lines as the analogous theorem in [12].

Theorem 7.4. Let $G, T_{0}, A(t), t \in G$, be as before. Let $U(t)$ depend only on t. Let $S$ be defined as before, $\{(t, y, u) \mid t \in G, y \in A(t), u \in U(t)\}$. Let $f$ : $S \rightarrow B$ and $g: S \rightarrow Z$ satisfy property (C). Let $\tilde{Q}(t, y), y \in A(t)$ be defined as before and be closed and convex for $t \in G-T_{0}$. Let $\xi(t), y(t), \xi_{k}(t), y_{k}(t)$, $\eta_{k}(t), u_{k}(t), \theta(t), \theta_{k}(t), t \in G, k=1,2, \ldots$, be measurable a.e. finite functions, $\eta_{k} \in L_{1}(G, Z), \xi, \xi_{k} \in L_{1}(G, B), \theta, \theta_{k} \in L_{1}\left(G, E^{1}\right), y_{k}(t) \in A(t)$, $u_{k}(t) \in U(t)$,

$$
\xi_{k}(t)=f\left(t, y_{k}(t), u_{k}(t)\right), \quad \eta_{k}(t)=g\left(t, y_{k}(t), u_{k}(t)\right)
$$

and let

$$
\tau_{k}(t)=g\left(t, y_{k}(t), u_{k}(t)\right)-g\left(t, y(t), u_{k}(t)\right)
$$

and

$$
\delta_{k}(t)=f\left(t, y_{k}(t), u_{k}(t)\right)-f\left(t, y(t), u_{k}(t)\right), \quad t \in G, k=1,2, \ldots
$$

We shall assume that $\Lambda \subset\{z \mid a z \geqslant \varepsilon\|a\|\|z\|\}$ for some $0<\varepsilon<1$ and $a \in-\Lambda^{*}$, and that

(i) $-\infty<i=\lim _{\inf _{k \rightarrow \infty} \int} a \eta_{k}(t) d \mu<\infty$; 
(ii) $\xi_{k} \rightarrow \xi$ weakly in $L_{1}(G, B), \theta_{k} \rightarrow \theta$ weakly in $L_{1}\left(G, E^{1}\right), \tau_{k} \rightarrow 0, \delta_{k} \rightarrow 0$, and $y_{k} \rightarrow y$ in measure in $G$ as $k \rightarrow \infty$;

(iii) $a \eta_{k}(t)>\theta_{k}(t), \mu$-a.e. in $G$ and $k=1,2, \ldots$

Then there exists a measurable function $u(t), t \in G$, such that $y(t) \in A(t)$, $u(t) \in U(t), \xi(t)=f(t, y(t), u(t))$, and if $\eta(t)=g(t, y(t), u(t))$, then $\int_{G} a \eta(t) d \mu$ exists and is $<i$. If condition $\left(\alpha_{0}\right)$ is also satisfied then $\eta \in$ $L_{1}(G, Z)$.

REMARK 7.3. It is known that if $\tilde{f}$ satisfies property (C), if $U$ is a Banach space, if $u_{k}(t), t \in G, k=1,2, \ldots$, belong to a norm bounded subset of $L_{p}(G, U)$ for some $p, 1<p<\infty$, then $\delta_{k} \rightarrow 0$ and $\tau_{k} \rightarrow 0$ in measure, and this particular hypothesis in the above theorem is automatically satisfied. For a proof of the above statement, we refer to Cesari and Suryanarayana [12]. If $y_{k}$ are also known to be $L_{p}$-bounded for some $p$, then the above statement is obtained from Lemma (2.1) of Krasnoselskii [21]. This special case where $u_{k}$ and $y_{k}$ are both $L_{p}$-bounded and $Z$ is the set of reals was considered by Berkovitz [1]. Let $T(t, y, z)=\operatorname{Inf}[\eta \mid(\eta, z) \in \bar{Q}(t, y)$ for some $z]$. If we use suitable selection theorems (such as Castaing's) instead of the McShaneWarfield lemma, then property (C) can be modified as follows: $T(t, y, z)$ is lower semicontinuous in $(y, z)$ for every $t$, and measurable in $t$ for every $(y, z)$ (cf. [35]). This is equivalent to a recent result of Olech [25]. Thus, the present paper extends these results to Pareto problems. In [35] Suryanarayana proved that, if $\Lambda$ is a polyhedral cone and $Z$ is finite dimensional, then, by suitable slight changes in the hypotheses, the conclusions of 6.1, 7.1, 7.3, 7.4 are valid for every $\alpha \in-\Lambda^{*}$. Finally, Cesari and Suryanarayana [34] proved that, in case where $B$ and $Z$ are finite dimensional, Theorem 7.3 is valid with property $(\mathrm{Q})$ replaced by mere property $(\mathrm{K})$ of the sets $\bar{Q}(t, y)$, and Theorem 7.4 holds without the property concerning $\delta_{k}$. Analogous remark holds for the other theorems.

8. Existence theorems for Pareto-Lagrange problems. We shall prove in this section existence theorems for control problems where the $Z$-valued cost functional is a Bochner integral on $G$. We need the lower closure theorems of \$7. If the cost functional is a Pettis integral one can use the lower closure theorems of Cesari [5], [6] for scalar valued functionals.

We shall follow the notations of $\$ 5$. Let $Z$ be a reflexive Banach space and let $\Lambda$ be a closed convex cone in $Z$ with the angle property; that is, there exist $\varepsilon$ real, with $0<\varepsilon<1$ and $a \in-\Lambda^{*}$ such that $\Lambda \subset\{z \mid a z>\varepsilon\|a\|\|z\|\}$. Let $g(t, y, u)$ be a function defined on $S$ with values in $Z$ or $g: S \rightarrow Z$. We assume that $A, S$ and the functions $f$ and $g$ satisfy Carathéodory conditions. We shall consider the sets

$$
\tilde{Q}(t, y)=\left\{\left(z^{0}, z\right) \in Z \times B \mid z^{0}=g(t, y, u), z=f(t, y, u), u \in U(t, y)\right\}
$$


and

$$
\begin{aligned}
& \tilde{Q}(t, y)=\tilde{Q}(t, y)+\Lambda=\left\{\left(z^{0}, z\right) \in Z \times B \mid z^{0} \in g(t, y, u)+\Lambda,\right. \\
& z=f(t, y, u), u \in U(t, y)\} .
\end{aligned}
$$

We say that a pair $(x, u), x \in X_{0}, u \in \mu(G, U)$, is admissible provided

$$
(M x)(t) \in A(t), \quad u(t) \in U(t, M x(t)), \quad L x(t)=f(t, M x(t), u(t)),
$$

and

$$
\|g(\cdot, M x(\cdot), u(\cdot))\| \in L_{1}\left(G, E^{1}, \mu\right) .
$$

Thus, the $Z$-valued (Bochner) integral

$$
I[x, u]=\int_{G} g(t, M x(t), u(t)) d \mu
$$

has a finite value in $Z$ for every admissible $(x, u)$. We shall consider a given class $\Omega$ of admissible pairs as in $\$ 5$, and thus $I[x, u]$ is a $Z$-valued functional defined on $\Omega$.

As in $\$ 5$, we denote by $\{x\}_{\Omega}$ the collection $\left\{x \in X_{0},(x, u) \in \Omega\right.$, for some $u$, so that $\{x\}_{\Omega} \subset X_{0} \subset X$. We shall say that the class $\Omega$ is closed (with respect to the Lagrange problem under consideration) provided, whenever $\left(x_{k}, u_{k}\right) \in \Omega, k=1,2, \ldots, x_{k} \rightarrow x$ in $(X, \tau)$ as $k \rightarrow \infty, x \in X_{0}$, and there is some $u \in \mu(G, U)$ such that $(x, u)$ is admissible, then there is also some $\bar{u} \in \mu(G, U)$ such that $(x, \bar{u}) \in \Omega$, and $I[x, \bar{u}]<_{\Lambda} I[x, u]$.

REMARK 8.1. For a fixed $\lambda^{*} \in \Lambda^{*}$, one may consider the sets

$$
-\lambda^{*} \tilde{Q}(t, y)=\left[(\mathscr{Z}, z) \in E^{1} \times B \mid \mathscr{Z}=-\lambda^{*} z^{0},\left(z^{0}, z\right) \in \tilde{Q}(t, y)\right]
$$

and the real valued functional

$$
-\lambda^{*} I=\int_{G}-\lambda^{*} g(t, M x(t), u(t)) d \mu .
$$

In this case, we may define a class $\Omega$ to be closed with respect to $\lambda^{*}$ as follows. Whenever $\left(x_{k}, u_{k}\right) \in \Omega, k=1,2, \ldots, x_{k} \rightarrow x$ in $(X, \tau)$ as $k \rightarrow \infty$, $x \in X_{0}$, and there is some $u \in \mu(G, U)$ such that $(x, u)$ is admissible, then there is also some $\bar{u} \in \mu(G, U)$ with $(x, \bar{u}) \in \Omega$ and $-\lambda^{*} I[x, \bar{u}]<$ $-\lambda * I[x, u]$.

One may require the existence of such $\bar{u}$ only for those sequences $\left(x_{k}, u_{k}\right)$ and the element $(x, u)$ with $-\lambda^{*} I[x, u] \leqslant \lim$ inf $-\lambda^{*} I\left[x_{k}, u_{k}\right]$.

We say, as in $\$ 6$, that a condition $\left(\alpha_{0}\right)$ holds if there is a $Z$-valued function $\phi(t), t \in G, \phi(t) \in Z$, with $\|\phi(t)\| \in L_{1}(G)$ such that $\phi(t)<_{\Lambda} g(t, y, u)$ for all $(t, y, u) \in S$.

REMARK 8.2. It is of interest to note that if $\Lambda$ is a closed convex cone in a 
Banach space $Z$ and if $f \in L_{1}(G, Z)$ with $f(t) \in \Lambda$, then $\int_{G} f(t) d \mu \in \Lambda$. Indeed, this is true for simple functions and $\int_{G} f(t) d \mu$ is defined as the limit of integrals of simple functions.

THEOREM 8.1. EXISTENCE THEOREM (for Pareto-Lagrange problems). Let $Z$ be a reflexive Banach space over the reals, $\Lambda$ a closed convex cone in $Z$, and $\Lambda^{*}$ the polar of $\Lambda$, such that $Z, \Lambda$ satisfy the angle property, so that $\Lambda \subset\{z \in Z \mid a z$ $>\varepsilon\|a\|\|z\|\}$ for some $\varepsilon$ real and $a \in Z^{*}$ with $0<\varepsilon<1$ and $-a \in$ int $\Lambda^{*} \neq$ $\varnothing$. Let $A, G, S, f, g$ satisfy conditions $(C)$ and $\left(\alpha_{0}\right)$. Let us assume that $\Omega$ is not empty and closed, and that $\{x\}_{\Omega}$ is sequentially relatively compact in $(X, \tau)$. Let us assume that both operators $L$ and $M$ have the closure property in $X_{0}$, and that at least one of them has the closure graph property in $X_{0}$. Finally, let us require the condition (AQ̃) $L: X_{0} \rightarrow L_{1}(G, B)$ has the weak convergence property relative to $\{x\}_{\Omega} ; M: X_{0} \rightarrow \mu(G, Y)$ has the convergence in measure property relative to $\{x\}_{\Omega}$; and, for $\mu$-almost all $t \in G$, the sets $\bar{Q}(t, y)=\Phi(t, y)+\Lambda$ are closed, convex, and have property $(Q)$ with respect to $y$ in $A(t)$. Then the $Z$-valued functional $I[x, u]$ defined by $(*)$ has at least one Pareto minimum in 及.

Mere property $(\mathrm{K})$ suffices, instead of property $(\mathrm{Q})$, if the space $B$ and $Z$ are finite dimensional (cf. Remark 7.3).

Proof. Let $\mathbb{Q}=\{I[x, u](x, u) \in \Omega\}$. Condition $\left(\alpha_{0}\right)$ and Remark 8.2 imply that $Q$ is bounded below. Indeed, there is a $\phi \in L_{1}(G, Z)$ such that $\phi(t)<_{\Lambda} g(t, y, u)$ or $g(t, M x(t), u(t))-\phi(t) \in \Lambda$ and, hence, $I[x, u]-$ $\int_{G} \phi(t) d \mu \in \Lambda$ for every admissible $[x, u]$. Thus, $\mathbb{Q} \subset c+\Lambda$ with $c=$ $\int_{G} \phi(t) d \mu$. Also $\mathbb{Q}$ is nonempty since $\Omega$ is. Just as for Mayer problems let us consider the set of real numbers $\{\mathscr{Z}=a z \mid z \in \mathbb{Q}\}$, where $a$ is the element of $Z^{*}$ appearing in the angle property of $\Lambda$. This set of real numbers is bounded below and nonempty. Hence, its infimum $j$ is finite. Let $z_{k} \in \mathbb{Q}$ be chosen such that $a z_{k} \rightarrow j$ as $k \rightarrow \infty$. If $z_{k}=I\left[x_{k}, u_{k}\right]$ with $x_{k} \in\{x\}_{\Omega}, k=1,2, \ldots$, then by weak sequential compactness of $\{x\}_{\Omega}$ (which is assumed) there exist a subsequence, say still $[k]$, and an element $x \in X$ such that $x_{k} \rightarrow x$ in $(X, \tau)$. Because of assumed convergence properties of $L$ and $M$, there is a subsequence, say still $k$, such that $L x_{k} \rightarrow y$ weakly in $L_{1}(G, B)$ for some $y \in L_{1}(G, B)$. Also $M x_{k} \rightarrow z$ for some $z \in \mu(G, B)$. By extracting a subsequence if necessary, we may assume that $M x_{k}(t) \rightarrow M x(t) \mu$-a.e. in $G$. Because of the closure properties of $L$ and $M$ we have $L x=y, M x=z$ and by the closed graph property, $x \in X_{0}$. By lower closure theorem 7.3 there is some $\bar{u}(t), t \in G, \bar{u} \in \mu(G, U)$-which, of course, may depend on $a$-such that $(x, \bar{u})$ is admissible. By the definition of closedness, and since $\Omega$ is closed, there is some element $u \in \mu(G, U)$-which again may depend on $a$-such that $(x, u) \in \Omega, x \in\{x\}_{\Omega}$ and 


$$
\mathscr{Z}=a I[x, u]<a I[x, \bar{u}]<\liminf _{k \rightarrow \infty} a I\left[x_{k}, u_{k}\right]=\lim _{k \rightarrow \infty} a z_{k}=j .
$$

But, since $x \in\{x\}_{\Omega}$ and, thus, $z=I[x, u] \in \mathbb{Q}$, we have $\mathscr{Z}=a z \geqslant j$ and, consequently, $\mathscr{Z}=a z=j$. Since by Remark 4.3, $-a \in$ int $\Lambda^{*}$, Lemma 3.1 and Remark 3.2 apply, and we see that $z=I[x, u]$ is $\Lambda$-Pareto optimal for the $\mathbb{Q}$ in $Z$, or that $(x, u)$ is $\Lambda$-Pareto optimal for the $Z$-valued functional $I[x, u]$ in $\Omega$. The theorem is proved.

REMARK 8.3. Just as for Mayer problems, we could use variants of the lower closure theorems mentioned above and obtain the existence of optimal solutions under a variety of alternate hypotheses, such as (AQ*), (AP), and (Fp) of Remark 6.1, with sets $Q(t, y)$ replaced by $\tilde{Q}(t, y)$. For details of these alternate hypotheses, we also refer to Cesari [5] and Cesari and Suryanarayana [13], [34].

We wish to single out, however, a particular class of Lagrange problems, where the minimizing sequence has a certain property (D) (see [11] which allows us to make no explicit seminormality requirements on the relevant sets. We use the lower closure theorem 7.4 and Remark 7.3.

THEOREM 8.2. Same as in Theorem 8.1 except for the following changes: $U$ is $a$ Banach space and $(x, u) \in \Omega$ implies that $u$ belongs to a fixed $L_{p}$-bounded set for some $p, 1<p<\infty$. The requirement of property $(Q)$ of the sets $\widetilde{Q}(t, y)$, is omitted.

We shall now state an important corollary (8.3) of the above theorem applied to ordinary differential equations. First we introduce the needed notations.

Let $A$ be a closed connected subset of the $t x$-space $E^{1} \times E^{n}$ whose projection on the $t$-axis is the finite interval $t_{0}<t<T$. For each $(t, x) \in A$, let $U(t, x)$ be a closed subset of the $u$-space $E^{m}$. Let $g(t, x, u)=$ $\left(g_{1}, \ldots, g_{N}\right), f(t, x, u)=\left(f_{1}, \ldots, f_{n}\right)$ be continuous vector functions on the set $S=\{(t, x, u) \mid(t, x) \in A, u \in U(t, x)\}$. Let $B_{1}, B_{2}$ denote given closed subsets of $A$, one of which is compact.

Let us consider the problem of Pareto optimization (with respect to the positive cone $\left.\Lambda=\left\{\left(\lambda_{1}, \ldots, \lambda_{N}\right) \mid \lambda_{i}>0, i=1, \ldots, N\right\} \subset E^{N}\right)$ for the cost functional $I[x, u]=\left(I_{1}, \ldots, I_{N}\right)$, where

$$
I_{j}[x, u]=\int_{t_{1}}^{t_{2}} g_{j}(t, x(t), u(t)) d t, \quad j=1, \ldots, N .
$$

We shall consider this problem in the class $\Omega$ of all pairs $x(t), u(t)$, $t_{1}<t<t_{2}, x \mathrm{AC}$ (absolutely continuous), $u$ measurable in $\left[t_{1}, t_{2}\right]$ satisfying the differential system

$$
d x / d t=f(t, x(t), u(t)), \quad t \in\left[t_{1}, t_{2}\right] \text { (a.e.), }
$$


the constraints

$$
(t, x(t)) \in A, \quad u(t) \in U(t, x(t)),
$$

and the boundary conditions

$$
\left(t_{1}, x\left(t_{1}\right)\right) \in B_{1} \text { and }\left(t_{2}, x\left(t_{2}\right)\right) \in B_{2} .
$$

In order to state an existence result, we introduce the sets

$$
\begin{array}{r}
\tilde{Q}(t, x)=\left[\left(\mathscr{L}^{1}, \ldots, \mathscr{Z}^{N}, z^{1}, \ldots, z^{n}\right) \mathscr{Q}^{j}>g_{j}(t, x, u), j=1, \ldots, N,\right. \\
\left.z^{i}=f_{i}(t, x, u), i=1, \ldots, n, u \in U(t, x)\right],
\end{array}
$$

for each $(t, x) \in A$.

THEOREM 8.3. With the above notation, let $S$ be closed and $\Omega$ be nonempty. Assume that there is a continuous scalar function $\Phi(\zeta), 0<\zeta<+\infty$, with $\Phi(\zeta) / \zeta \rightarrow+\infty$ as $\zeta \rightarrow+\infty$, and an index $j_{0}$ with $1<j_{0}<N$ such that $g_{j_{0}}(t, x, u)>\Phi(|f(t, x, u)|)$ for all $(t, x, u) \in S$. Let the sets $\bar{Q}(t, x)$ be closed and convex (and therefore by the slow growth condition just assumed, these sets $\tilde{Q}(t, x)$ will, for each $\bar{t} \in\left[t_{0}, T\right]$, satisfy property $(\mathrm{Q})$ with respect to $x \in A(\bar{t})$ $=\{x \mid(\bar{t}, x) \in A\}$; see Cesari [9]). Finally, let us assume that there are $L_{1^{-}}$ integrable scalar functions $\phi_{j}(t), t \in\left[t_{0}, T\right]$, such that $g_{j}(t, x, u)>\phi_{j}(t)$ for all $(t, x, u) \in S, j=1, \ldots, N, j \neq j_{0}$.

Then there is at least one $\Lambda$-Pareto optimal solution $[x, u]$ for the cost functional $I$ in the class $\Omega$.

Remark 8.4. We may obtain the Pareto optimum in a smaller class $\Omega^{\prime} \subset \Omega$ provided $\Omega^{\prime}$ is nonempty and is "closed" in the sense of \$7. A growth condition on $g_{j_{0}}$ is required to guarantee the equiabsolute integrability of the $x_{k}^{\prime} \in L_{1}$.

We shall now obtain as a corollary an existence theorem (8.4) for optimal solutions for systems described by total differential equations. The following notations are needed.

Let $G$ be a bounded open subset of the $t$-space $E^{\nu}$ with regular Sobolev boundary. For the sake of simplicity we shall assume that $G$ is of class $K_{l}$, $l>1$, that is, $G$ is bounded by a surface $\Gamma$ which can be decomposed into finite numbers of manifolds $\Gamma_{1}, \ldots, \Gamma_{J}$ of dimension $\nu-1$ (and corresponding boundaries), each $\Gamma_{j}$ having the property that it can be mapped into a hyperplane $\pi_{j}$ by means of a transformation of coordinates $\sigma_{j}$ defined on a part $G_{j}$ of $G$ and continuous with continuous derivatives up to order $l$, for $j=1, \ldots, J$.

For each $t \in \mathrm{cl}(G)$, let $A(t)$ be a nonempty subset of the $x$-space $E^{n}$, and we shall assume that the set $A$ of all $(t, x) \in E^{\nu} \times E^{n}$ with $t \in \operatorname{cl}(G)$ and $x \in A(t)$ is closed. For each $(t, x) \in A$, let $U(t, x)$ be a nonempty closed subset of the $u$-space $E^{m}$. Let $S$ be the set of all $(t, x, u) \in E^{\nu} \times E^{n} \times E^{m}$ 
with $u \in U(t, x)$ and $(t, x) \in A$. For each $i=1, \ldots, n$, let $\{\alpha\}_{i}$ be a given finite system of indices $\alpha=\left(\alpha_{1}, \ldots, \alpha_{v}\right), 0<|\alpha|<l_{i}<l$, where $|\alpha|=\alpha_{1}$ $+\cdots+\alpha_{p}$. Let $N_{1}$ be the total number of elements $\alpha \in\{\alpha\}_{i}, i=1, \ldots, n$. Let $g=\left(g_{1}, \ldots, g_{N_{0}}\right)$ and $f=\left(f_{i \alpha}, \alpha \in\{\alpha\}_{i}, i=1, \ldots, n\right)$ be continuous vector valued functions on $S$. Let

$$
\begin{aligned}
\tilde{Q}(t, x)=\left\{(\mathscr{Z}, z) \in E^{N_{0}} \times E^{N_{1}} \mid \mathscr{Z}^{j}>g_{j}(t, x, u), j\right. & =1,2, \ldots, N_{0} \\
z & =f(t, x, u), u \in U(t, x)\} .
\end{aligned}
$$

We shall impose boundary conditions (B) on the trajectories $x^{i}(t)$ and their derivatives $D^{\alpha} x^{i}(t)$ in such a way that the following closure properties $\left(\mathrm{P}_{1}\right)$ and $\left(P_{2}\right)$ are valid:

$\left(\mathrm{P}_{1}\right)$ If $x(t)=\left(x^{1}, \ldots, x^{n}\right), x_{k}(t)=\left(x_{k}^{1}, \ldots, x_{k}^{n}\right), t \in G, k=1,2, \ldots$, are vector functions whose components $x^{i}, x_{k}^{i}$ belong to the Sobolev class $W_{p_{1}}^{l_{1}}(G)$, if $D^{\beta} x_{k}^{i}(t) \rightarrow D^{\beta} x^{i}(t)$ as $k \rightarrow \infty$ strongly in $L_{p_{1}}(G)$ for every $\beta=$

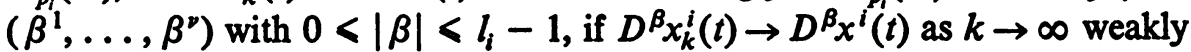
in $L_{p_{i}}(G)$ for every $\beta$ with $|\beta|=l_{i}$, and if the boundary values $\phi_{k_{\alpha}}^{i}$ of $x_{k}^{i}(t)$, $i=1, \ldots, n, 0<|\alpha|<l_{i}-1$, on $\partial G$ satisfy the boundary conditions (B), then the boundary values $\phi_{\alpha}^{i}$ of $x^{i}(t), i=1, \ldots, n, 0<|\alpha|<l_{i}-1$, on $\partial G$ satisfy the boundary conditions (B).

$\left(\mathrm{P}_{2}\right)$ If $x(t)=\left(x^{1}, \ldots, x^{n}\right), t \in G$, is any vector function satisfying boundary conditions (B) and whose components $x^{i}(t) \in W_{p_{1}}^{h}(G), p_{i}>1$, $1<l_{i}<l, \int_{G}\left|D^{\beta} x^{i}(t)\right|^{p_{i} d t}<M_{i_{\beta}}$ for all $\beta=\left(\beta_{1}, \ldots, \beta_{\nu}\right)$ with $|\beta|=l_{i}, i=$ $1, \ldots, n$, and constants $M_{i_{\beta}}$, then there are constants $M_{i_{\alpha}}$ such that $\int_{G}\left|D^{\alpha} x^{i}(t)\right| d t<M_{i_{\alpha}}$ for all $\alpha=\left(\alpha_{1}, \ldots, \alpha_{v}\right)$ with $0<|\alpha|<l_{i}-1, i=$ $1, \ldots, n$, where the constants $M_{i_{\alpha}}$ depend only on $p_{i}, \nu$, all $M_{i_{\beta}}, G$ and the boundary conditions (B), but not on the vector function $x(t)$ above.

Let $\Omega$ be a nonempty closed class of pairs $x(t)=\left(x^{1}, \ldots, x^{n}\right), u(t)=$ $\left(u^{1}, \ldots, u^{m}\right), t \in G, x^{i}(t) \in W_{p_{i}}^{l}(G), 1<l_{i}<l, p_{i}>1, i=1, \ldots, n, u^{m}(t)$ measurable in $G, j=1, \ldots, m$, satisfying (a) the constraints $(t, x(t)) \in A$, $u(t) \in U(t, x(t))$ a.e. in $G$, (b) the system of partial differential equations $D^{\alpha} x^{i}(t)=f_{i \alpha}(t, x(t), u(t))$ a.e. in $G, \alpha \in\{\alpha\}_{i}, i=1, \ldots, n$, (c) the boundary conditions (B) on the boundary $\partial G$ of $G$ (satisfying closure properties $\left(\mathrm{P}_{1}\right)$ and $\left.\left(\mathrm{P}_{2}\right)\right)$ on the boundary values of $x^{i}(t)$ and $D^{\beta} x^{i}(t)$ for $0<|\beta|<l_{i}-1, i=1, \ldots, n$, and (d) the system of inequalities $\int_{G}\left|D^{\beta} x^{i}(t)\right|^{p_{i}} d t<N_{i_{\beta}}$ for all $\beta$ with $|\beta|=l_{i}, \beta \notin\{\alpha\}_{i}, i=1, \ldots, n$, where $N_{i_{\beta}}$ are given constants, and finally (e) $g_{j}(t, x(t), u(t)) \in L_{1}(G), j=$ $1, \ldots, N_{0}$.

In view of the last lines of Remark 7.3, we are now in a position to state

THEOREM 8.4. With the above notations, if $S$ is closed, if the sets $\tilde{Q}(t, x)$, $x \in A(t)$ satisfy property $(\mathrm{K})$ with respect to $x$ only, for each $t \in G$, if there 
exist functions $\phi_{j} \in L_{1}(G)$ with $g_{j}(t, x, u) \geqslant \phi_{j}(t), j=1, \ldots, N_{0}$, and $(t, x, u)$ $\in S$, and if for all $(x, u) \in \Omega$ with $\int_{G} g_{j_{0}}(t, x(t), u(t)) \leqslant L_{0}, j_{0}$ fixed, $1 \leqslant j_{0} \leqslant$ $N_{0}, L_{0}$ a constant, we also have $\int_{G}\left|D^{\alpha} x^{i}(t)\right|^{p_{i}} d t \leqslant L_{i \alpha}, \alpha \in\{\alpha\}_{i}, i=$ $1, \ldots, n$, with $L_{i \alpha}$ depending only on $L_{0}, \Omega, p_{i}, l_{i}$ and on the boundary conditions (B), then the vector valued cost functional $I[x, u]=\left(I_{1}, \ldots, I_{N_{0}}\right)$, with $I_{j}=$ $\int_{G} g_{j}(t, x(t), u(t)) d t$, has a Pareto minimum in $\Omega$, that is, there is $a(\bar{x}, \bar{u}) \in \Omega$, such that $I_{j}[x, u]<I_{j}[x, u], j=1, \ldots, N_{0}$, for some $[x, u] \in \Omega$, implies $I[x, u]=I[\bar{x}, \bar{u}]$.

REMARK. Note that if we impose slow growth conditions as in Theorem 8.3, then we ensure property $(Q)$ of the sets $\bar{Q}$ above. If we only know that $|g(t, x, u)| \rightarrow \infty$ as $|u| \rightarrow \infty$ uniformly in every compact subset of $A(t)$, then the same sets certainly have the required property $(K)$.

Example 1 (See Cesari and Cowles [10].) Let $G$ be a subset of the $(t, \tau)$ space $R^{\nu+1}, \tau=\left(\tau^{1}, \ldots, \tau^{\nu}\right)$, of the form $G=(0, T) \times G^{\prime}$, where $G^{\prime}$ is an open bounded connected subset of $R^{\nu}$ of class $K_{l}$. The boundary $\Gamma=\partial G$ is made up of three parts: $\Gamma_{1}=\{0\} \times \operatorname{cl~} G^{\prime}, \Gamma_{2}=[0, T] \times \partial G^{\prime}, \Gamma_{3}=\{T\} \times$ cl $G^{\prime}$. On $\Gamma_{1}$ and $\Gamma_{3}$ we have the Lebesgue $\nu$-dimensional measure or $\|_{\nu}$ (and we shall use the symbol $d \tau$ in integration). In $\Gamma_{2}$ we have the product measure $\sigma=\mid \|_{1} \times \mu$ of the one-dimensional measure on $[0, T]$ and of hyperarea $\mu$ on the boundary $\partial G^{\prime}$ of $G^{\prime}$ (and we shall use the symbol $d t d \mu$ in integration). Given a function $x$ in $G$, we shall denote by $\gamma x$ the boundary values of $x$ on $\Gamma=\partial G$, and, specifically, we shall denote by $\gamma_{i} x$ the boundary values of $x$ on $\Gamma_{i}, i=1,2,3$. We shall denote by $T, T_{i}^{0}$ the families of all measurable functions on $G, \Gamma_{i}, i=1,2,3$, respectively.

Let $S_{p}^{l}(G), 1<p<+\infty, l \geqslant 1$, be the space of all real valued functions $x(t, \tau),(t, \tau) \in G$, such that $\partial x / \partial t$ and all $D_{\tau}^{\alpha} s, \alpha=\left(\alpha_{1}, \ldots, \alpha_{\nu}\right), 0<|\alpha|<$ $l$, exist as generalized derivatives and are all in $L_{p}(G)$. We shall make $S_{p}^{l}(G)$ a Banach space by means of the norm

$$
\|\dot{x}\|=\|x\|_{S_{p}^{\prime}(G)}=\left(\int_{G}\left|\frac{\partial x}{\partial t}\right|^{p} d t d \tau\right)^{1 / p}+\sum_{0<|\alpha|<l}\left(\int_{G}\left|D_{\tau}^{\alpha} x\right|^{p} d t d \tau\right)^{1 / p} .
$$

These spaces $S_{p}^{l}(G)$ have weak compactness properties similar to those for Sobolev spaces. Each element $x \in S_{p}^{l}(G)$ possesses the boundary values $\gamma_{1} x \in L_{p}\left(\Gamma_{1}\right), \gamma_{3} x \in L_{p}\left(\Gamma_{3}\right)$ on $\Gamma_{1}, \Gamma_{3}$, respectively, and all $\gamma_{2} D_{\tau}^{\alpha} x \in L_{p}\left(\Gamma_{2}\right)$, $0<|\alpha|<l-1$, on $\Gamma_{2}$. We are concerned with the Pareto minimum of the vector valued functional $\left(I_{1}, I_{2}, I_{3}\right)$ with

$$
\begin{aligned}
& I_{1}=\int_{G} f_{0}(t, \tau, M x(t, \tau), u(t, \tau)) d t d \tau \\
& I_{2}=\int_{\Gamma_{2}} g_{0}\left(t, \tau,(K x)(t, \tau), v_{2}(t, \tau)\right) d t d \mu \\
& I_{3}=\int_{\Gamma_{3}} g_{0}\left(t,(K x)(T, \tau), v_{3}(\tau)\right) d \tau
\end{aligned}
$$


Here $M x=\left(x, \nabla_{\tau} x\right)$ and $K x=\partial x / \partial n$ on $\Gamma_{2}$ and $K x=x$ on $\Gamma_{3}$. Also,

and

$$
f_{0}=t^{2}+|\tau|^{2}+x^{2}+\left|\nabla_{\tau} x\right|^{2}+|u|-1>-1,
$$

$$
g_{0}=(\partial x / \partial n)^{2}+v_{2}^{2} \geqslant 0 \text { on } \Gamma_{2}, \quad g_{0}=x^{2}-1 \geqslant-1 \text { on } \Gamma_{3} .
$$

We consider state equations of the form

$$
\begin{aligned}
& (\mathscr{L} x)(t, \tau)=f(t, \tau,(M x)(t, \tau), u(t, \tau)) \quad \text { a.e. in } G, \\
& (\mathscr{G} x)(t, \tau)=g\left(t, \tau,(K x)(t, \tau), v_{2}(t, \tau)\right) \quad \sigma \text {-a.e. in } \Gamma_{2}
\end{aligned}
$$

with

$$
\mathfrak{L}_{x}=\frac{\partial x}{\partial t}-\sum_{i=1}^{\nu} \frac{\partial^{2} x}{\left(\partial \tau^{i}\right)^{2}} \quad \text { and } \mathscr{G}_{x}=\sum_{i=1}^{\nu} a_{i}(t, \tau) \gamma_{2}\left(\frac{\partial x}{\partial \tau^{i}}\right),
$$

where $a_{i}$ are given elements of $L_{2}\left(\mathrm{\Gamma}_{2}\right)$. Also,

$$
f=t+\sum_{i} \tau^{i}+x+\sum_{i} \frac{\partial x}{\partial \tau^{i}}+u+2^{-1}|u|, \quad g=x \frac{\partial x}{\partial n}+v_{2} \quad \text { on } \Gamma_{2} .
$$

Let $\Omega$ be the set of all admissible systems $x \in S_{p}^{l}(G), u \in T, v_{2} \in T_{2}^{0}$ with $\|x\|_{S_{p}^{2}(G)}<N$ for sufficiently large $N$.

Here $Z=R^{3}$ and $\Lambda=\left\{\left(\lambda_{1}, \lambda_{2}, \lambda_{3}\right) \mid \lambda_{i} \geqslant 0, i=1,2,3\right\}$. Clearly, $\Lambda$ is a closed convex cone and is acute and satisfies property $(\pi)$ with

$$
\lambda^{*}=(-1,-1,-1) \in \operatorname{int} \Lambda^{*}=\operatorname{int}\left\{\left(\lambda_{1}, \lambda_{2}, \lambda_{3}\right) \mid \lambda_{i}<0, i=1,2,3\right\} .
$$

Due to weak compactness of spheres and imbedding theorems, it is seen that $M$ and $K$ have convergence in measure property while $\mathcal{L}$ and $G$ have weak convergence property. In fact, in this case, $x_{k} \rightarrow x$ weakly in $S_{p}^{2}(G)$ implies $\mathcal{L} x_{k} \rightarrow \mathcal{L} x$ weakly in $L_{1}(G), g x_{k} \rightarrow g x$ weakly in $L_{1}\left(\Gamma_{2}\right), M x_{k} \rightarrow M x$ in $\left(L_{1}(G)\right)^{p+1}$ and $K x_{k} \rightarrow K x$ in $L_{1}(\Gamma)$ strongly.

Here $U=\left\{\left(u, v_{2}\right) \mid u, v_{2}\right.$ real $\}$ and $\tilde{Q}=\left\{z_{1}^{0}>f_{0}, z_{2}^{0}>g_{0}, z_{3}^{0}>\left.g_{0}\right|_{\Gamma_{3}}, z_{1}=\right.$ $\left.f, z_{2}=g\right\}$, so that

$$
\begin{gathered}
\tilde{Q}\left(t, \tau, x, \nabla_{\tau} x\right)=\left\{z_{1}^{0}>t^{2}+|\tau|^{2}+x^{2}+\left|\nabla_{\tau} x\right|^{2}+|u|-1, z_{2}^{0}>\left(\frac{\partial x}{\partial n}\right)^{2}\right. \\
+v_{2}^{2}, z_{3}^{0}>x^{2}-1, z_{1}=t+\sum_{i} \tau^{i}+x+\sum_{i} \frac{\partial x}{\partial \tau^{i}} \\
\left.+u+2^{-1}|u|, z_{2}=x \frac{\partial x}{\partial n}+v_{2}, u, v_{2} \text { real }\right\} \\
=\left\{z_{1}^{0} \geqslant t^{2}+|\tau|^{2}+x^{2}+\left|\nabla_{\tau} x\right|^{2}-1, z_{2}^{0}>\left(\frac{\partial x}{\partial n}\right)^{2},\right. \\
\left.z_{3}^{0}>x^{2}-1, z_{1}, z_{2} \mid z_{1}, z_{2} \text { real }\right\},
\end{gathered}
$$

and property $(Q)$ is easily verified. 
EXAMPLE 2. Consider the problem of $\Lambda$-Pareto minimum of the functional $\left(\int_{0}^{\pi} x^{2}(t) d t, \int_{0}^{\pi} \exp (x(t)) d t\right)$ subject to the conditions $x^{\prime \prime \prime}=0, x(0)=1, x(\pi)=$ 1 , where $\Lambda=\{(a, b) \mid a>0, b>0, a, b$ real $\} \subset R^{2}$. Here, the problem is one of calculus of variations with no controls. The admissible trajectories (AC solutions) are $x(t)=a\left(t^{2}-\pi t\right)+1, a$ real. Thus,

$$
\begin{aligned}
I_{1} & =\int_{0}^{\pi} x^{2}(t) d t=\int_{0}^{\pi}\left[a^{2} t^{2}(t-\pi)^{2}+1+2 a t(t-\pi)\right] d t \\
& =a^{2}\left(3^{-1} \pi^{5}-2 \pi^{5} 4^{-1}+\pi^{5} 5^{-1}\right)+2 a\left(\pi^{3} 3^{-1}-\pi^{3} 2^{-1}\right)+\pi \\
& =a^{2}\left(\pi^{5} / 30\right)-a\left(\pi^{3} / 3\right)+\pi,
\end{aligned}
$$

which is minimum for $a=5 / \pi^{2}$. Thus, $x_{0}(t)=5 \pi^{-2}\left(t^{2}-\pi t\right)+1$ is a Pareto optimal solution. However $x(t) \stackrel{\text { def }}{=} t^{2}-\pi t+1<x_{0}(t)$ for all $t \in(0, \pi)$ so that

$$
I_{2}[x]=\int_{0}^{\pi} e^{x(t)} d t<I_{2}\left[x_{0}\right]=\int_{0}^{\pi} \exp \left(x_{0}(t)\right) d t
$$

Thus, $x_{0}(t)$ may not minimize the functionals simultaneously.

Example 3 [14, Example 1, p. 14]. Production scheduling problem. It is known that at time $t_{0}$ the production rate $p(t)$ is $p\left(t_{0}\right)=p_{0}$; it is required that at time $t_{1}$ the production rate be $p\left(t_{1}\right)=p_{1}$. The objective is to find the Pareto minimum (with respect to the positive cone, $\Lambda=\left\{\left(\lambda_{1}, \lambda_{2}\right) \mid \lambda_{1}>0, \lambda_{2}>0\right\}$ ) of the cost vector $\left(\int_{t_{0}}^{t_{1}} c_{1}, \int_{t_{0}}^{t_{1}} c_{2}\right)$; here $c_{1}$ is the machine cost, which is assumed to vary on the square of rate of change $\dot{p}(t)$ of the production rate; and $c_{2}$ is the labor cost associated with the change in the production rate-assumed to be given by $c_{2}=k t \dot{p}(t)$ where $k$ is a constant.

This is a free problem. Thus, $p(t)$ is the trajectory, $\dot{p}(t)$ is the control. We assume that there is a constant $a$ (possibly negative) such that $\dot{p}(t)>a$ for all $t \in\left[t_{0}, t_{1}\right]$. In this case the sets $\tilde{Q}(t, x)$ are $\left\{\left(\mathscr{Z}^{1}, \mathscr{L}^{2}, z\right) \mid \mathscr{Z}^{1}>u^{2}, \mathscr{Z}^{2}>t u, z\right.$ $=u, u>a\}$ for $t \in\left[t_{0}, t_{1}\right], x \in E^{1}$. For fixed $t$, these sets are fixed and, hence, have property $(Q)$ with respect to $x$.

Theorem 8.3 applies and the existence of at least one Pareto optimal solution is guaranteed.

EXAMPLe 4 [13, Examples 10, 12, pp. 70, 87]. It is desired to find a production rate $P(t)$ and an advertising rate $A(t)$ such that inventory level is changed from $I(0)=I^{0}$ to $I(T)=I^{1}$ in such a way that the cost $c_{T}=$ $\int_{0}^{T} k_{1} P^{2}(t) d t$ is minimized and the total sales $S_{T}=\int_{0}^{T} s(t) d t$ is maximized, subject to

$$
\begin{gathered}
d I / d t=P(t)-S(t), \quad I(0)=I^{0}, \quad I(T)=I^{1}, \\
d S / d t=-\alpha P(t)+\gamma A(t)-\left[\lambda+\gamma M^{-1} A(t)\right] S(t), \quad S(0)=S_{0},
\end{gathered}
$$

where $S(t)$ is the sales rate at time $t$, and the controls $P(t)$ and $A(t)$ are bounded, $\underline{P}<P(t)<\bar{P}$, and $\underline{A}<A(t)<\bar{A}$, and $\alpha, \gamma, M$ are constants. 
Once again Theorem 8.3 can be applied with the positive cone replaced by $\Lambda^{\prime}=\left\{\left(\lambda_{1}, \lambda_{2}\right) \mid \lambda_{1} \geqslant 0, \lambda_{2} \leqslant 0\right\}$.

Appendix. Here we shall present a second criterion for $\Lambda$-Pareto optimality of subsets $Q$ of a Banach space $Z$ with respect to a closed convex cone $\Lambda$ in $Z$. Given $\lambda^{*} \in \Lambda^{*}$, let us note, as in Yu [24], the set $\left\{a \lambda^{*}|a\rangle 0\right\}$ by $\left\langle\lambda^{*}\right\rangle$ and the set $\Lambda^{*}-\left\langle\lambda^{*}\right\rangle=\left\{\mu^{*}-a \lambda^{*} \mid a \geqslant 0, \mu^{*} \in \lambda^{*}\right\}$ by $\left\langle\lambda^{*}\right\rangle^{\perp}$. Thus any $\mu^{*} \in$ $\Lambda^{*}$ is of the form $\mu_{1}^{*}+\mu_{2}^{*}$ with $\mu_{1}^{*} \in\left\langle\lambda^{*}\right\rangle$ and $\mu_{2}^{*} \in\left\langle\lambda^{*}\right\rangle^{\perp}$. Given $\mathbb{Q} \subset Z$, $Q \neq \varnothing, z_{0} \in Z$ and $\lambda^{*} \in \Lambda^{*}$ let

$$
Y\left(\lambda^{*}, z_{0}\right)=\left\{z \in \mathbb{Q}\left|\mu^{*} z\right\rangle \mu^{*} z_{0} \text { for all } \mu^{*} \in\left\langle\lambda^{*}\right\rangle^{\perp}\right\}
$$

and

$$
M\left(\lambda^{*}\right)=\left\{z_{0} \in \mathrm{w}-\mathrm{cl}(\mathbb{Q}) \mid \lambda^{*} z>\lambda^{*} z_{0} \text { for all } z \neq z_{0}, z \in Y\left(\lambda^{*}, z_{0}\right)\right\} .
$$

LeMma 2.1. For each $\lambda^{*} \in \Lambda^{*}$, $\operatorname{Ext}_{\Lambda}(\mathscr{Q})=M\left(\lambda^{*}\right)$.

Proof. Let $z_{0} \in \operatorname{Ext}_{\Lambda}(\mathscr{Q})$ and $\lambda^{*} \in \Lambda^{*}$. Then $z_{0} \notin M\left(\lambda^{*}\right)$ implies $\lambda^{*} z_{0}<$ $\lambda^{*} z$ for some $z \neq z_{0}$ with $z \in Y\left(\lambda^{*}, z_{0}\right)$; that is, for some $z \neq z_{0}$ with $\mu^{*} z>\mu^{*} z_{0}$ for all $\mu^{*} \in\left\langle\lambda^{*}\right\rangle^{\perp}$. But then for any $\mu^{*} \in \Lambda^{*}, \mu^{*}=\mu_{1}^{*}+\mu_{2}^{*}$, with $\mu_{1}^{*} \in\left\langle\lambda^{*}\right\rangle^{\perp}, \mu_{2}^{*} \in\left\langle\lambda^{*}\right\rangle$ and $\mu^{*} z_{0}=\mu_{1}^{*} z_{0}+\mu_{2}^{*} z_{0}\left\langle\left(\mu_{1}^{*}+\mu_{2}^{*}\right) z=\mu^{*} z\right.$. Thus, for some $z \neq z_{0}, \mu^{*}\left(z_{0}-z\right)<0$ for all $\mu^{*} \in \Lambda^{*}$, and, hence, $z_{0} \in z+$ $\Lambda$ contrary to $\Lambda$-Pareto optimality of $z_{0}$.

Conversely, let for some $\lambda^{*}, z_{0} \in M\left(\lambda^{*}\right)$. Thus, $z_{0} \in \mathrm{w}-\mathrm{cl}(\mathfrak{Q})$ and $\lambda^{*} z_{0}>$ $\lambda^{*} z$ for all $z \in Y\left(\lambda^{*}, z_{0}\right), z \neq z_{0}$. Thus, given $z \in \mathbb{Q}, z \neq z_{0}$, either $z \in$ $Y\left(\lambda^{*}, z_{0}\right)$ and, hence, $\lambda^{*} z_{0}>\lambda^{*} z$ so that $z_{0} \notin z+\Lambda$, or $z \notin Y\left(\lambda^{*}, z_{0}\right)$ and, hence, there is a $\mu^{*} \in\left\langle\lambda^{*}\right\rangle^{\perp}$ with $\mu^{*} z_{0}<\mu^{*} z$ and $z_{0} \notin z+\Lambda$. Thus, $z_{0} \in$ $\operatorname{Ext}_{\Lambda}(\mathbb{Q})$.

Remark. For $Z=R^{l}$, the $l$-dimensional Euclidean space, and if $\Lambda$ is a polyhedral cone, then the above lemma reduces to Corollary 4.4 of $\mathrm{Yu}$ [15]. (See also Aubin [33].)

\section{REFERENCES}

1. L. D. Berkovitz, Lower closure and existence theorems in optimal control, Proc. Internat. Conf. Differential Equations, Academic Press, New York, 1975, pp. 26-39.

2. A. Blaquière (Editor), Topics in differential games, North-Holland, Amsterdam, 1973. 880.

3. N. Bourbaki, Espaces vectoriels topologiques, Chapters I, II, Hermann, Paris, 1955. MR 14,

4. L. Cesari, Existence theorems for weak and usual optimal solutions in Lagrange problems with unilateral constraints. I,II, Trans. Amer. Math. Soc. 124 (1966), 369-412; ibid.124 (1966), 413-430. MR 34 \#3392; 34 \#3393.

5. Geometric and analytic views in existence theorems for optimal control in Banach spaces. I: Distributed parameters; II: Distributed and boundary controls; III: Weak solutions, J. Optimization Theory Appl. 14 (1974), 505-520; ibid. 15 (1975), 467-497; ibid. 19 (1976), 185-214.

6. Lower semicontinuity and lower closure theorems without seminormality conditions, Ann. Mat. Pura Appl. (4) 48 (1974), 381-397. MR 49 \# 9705. 
7. Closure theorems for orientor fields and weak convergence, Arch. Rational Mech. Anal. 55 (1974), 332-356. MR 50 \#3081.

8. Soboleo spaces and multidimensional Lagrange problems of optimization, Ann. Scuola Norm. Sup. Pisa (3) 22 (1968), 193-227. MR 38 \# 1574.

9. Existence theorems for optimal controls of the Mayer type, SIAM J. Control 6 (1968), 517-552. MR 39 \#4722.

10. L. Cesari and D. E. Cowles, Existence theorems in multidimensional problems of optimization with distributed and boundary controls, Arch. Rational Mech. Anal. 46 (1972), 321-355. MR 49 \#1271.

11. L. Cesari and M. B. Suryanarayana, Closure theorems without seminormality conditions, J. Optimization Theory Appl. 15 (1975), 441-465.

12. __ Nemytsky's operators and lower closure theorems, J. Optimization Theory Appl. 19 (1976), 165-184.

13. __ Existence theorems for Pareto problems of optimization, Conf. Calculus of Variations, and Control Theory, Math. Research Center, Madison, Wis., 1975.

14. M. Connors and D. Teichrow, Optimal control of dynamic operations research models, Internat. Textbook, Scranton, Pa., 1967.

15. N. O. Dacunha and E. Polak, Constrained minimization under vector-valued criteria in linear topological spaces, Mathematical Theory of Control, Academic Press, New York, 1967, pp. 96-108. MR 41 \#2496.

16 M. M. Day, Normed linear spaces, Academic Press, New York, 1962. MR 26 \#2847.

17. G. Jameson, Ordered linear spaces, Lecture Notes in Math., vol.141, Springer-Verlag, Berlin and New York, 1970.

18. P. J. Kaiser, Existence theorems in the calculus of variations, Ph.D. thesis, Univ. of Michigan, 1973.

19. P. J. Kaiser and M. B. Suryanarayana, Orientor field equations in Banach spaces, J. Optimization Theory Appl. 19 (1976), 141-164.

20. V. L. Klee, Jr., Convex sets in linear spaces. I, II, Duke Math. J. 18 (1951), 443-446; ibid. 18 (1951), 875-883. MR 13, 354; 13, 849.

21. M. A. Krasnosel'skii, P. P. Zabreìko, E. I. Pustyl'nik and P. E. Sobolevskii, Integral operators in spaces of summable functions, "Nauka", Moscow, 1966. (Russian) MR 34 \#6568.

22. E. J. McShane and R. B. Warfield, On Filippov's implicit functions lemma, Proc. Amer. Math. Soc. 18 (1967), 41-47. MR 34 \#8399.

23. J. J. Moreau, Convexity and duality, Functional Analysis and Optimization, Academic Press, New York, 1966, pp. 145-169. MR 36 \#706.

24. C. Olech, Lexicographical order, range of integrals and "bang-bang" principle, Mathematical Theory of Control, Academic Press, New York, 1967, pp. 35-45. MR 40 \#7916.

25. _ Weak lower semicontinuity of integral functionals, J. Optimization Theory Appl. 19 (1976), 3-16.

26. V. Pareto, Course d'economie politique, Lausanne, Rouge, 1896.

27. S. Smale, Global analysis and economics, Dynamical Systems, Academic Press, New York, 1973. MR 49 \#6283.

28. M. B. Suryanarayana, Remarks on lower semicontinuity and lower closure, J. Optimization Theory Appl. 19 (1976), 125-140.

29. H. F. Weinberger, Conditions for local Pareto optima (to appear).

30. P. L. Yu, Cone convexity, cone extreme points, and nondominated solutions in decision problems with multiobjectives, J. Optimization Theory Appl. 14 (1974). MR 52 \#2628.

31. P. L. Yu and G. Leitmann, Nondominated decisions and cone convexity in dynamic multicriteria decision problems, J. Optimization Theory Appl. 14 (1974), 573-584. MR 52 \# 10072.

32. L. A. Zadeh, Optimality and nonscalar valued performance criteria, IEEE Trans. Automatic Control AC-8 (1963), 59-60.

33. J. P. Aubin, $A$ Pareto minimum principle, Differential Games and Related Topics (H. W. Kuhn and G. P. Szego, Editors), North-Holland, Amsterdam, 1971, pp. 147-175. 
34. L. Cesari and M. B. Suryanarayana, An existence theorem for Pareto problems, Nonlinear Analysis, Theory, Methods and Applications 2 (1978), 225-233.

35. M. B. Suryanarayana, Remarks on existence theorems for Pareto optimality, Dynamical Systems, a University of Florida International Symposium (A. R. Bednarek and L. Cesari, Editors), Academic Press, New York, 1977, pp. 335-347.

36. W. Stadler, A survey of multicriteria optimization, 1977 (preprint).

37. C. Olech, Existence theorems for optimal problems with vector valued cost function, Trans. Amer. Math. Soc. 136 (1969), 159-180.

Department of Mathematics, University of Michigan, ANn Arbor, Michigan 48109

Department of Mathematics, EAstern Michigan University, Ypsitanti, Michigan 48197 